\title{
Spectral convergence of manifold pairs
}

\author{
Karsten Fissmer and Ursula Hamenstädt*
}

\begin{abstract}
Let $\left(M_{i}, A_{i}\right)_{i}$ be pairs consisting of a complete Riemannian manifold $M_{i}$ and a nonempty closed subset $A_{i}$. Assume that the sequence $\left(M_{i}, A_{i}\right)_{i}$ converges in the Lipschitz topology to the pair $(M, A)$. We show that there is a number $c \geq 0$ which is determined by spectral properties of the ends of $M_{i}-A_{i}$ and such that the intersections with $[0, c)$ of the spectra of $M_{i}$ converge to the intersection with $[0, c)$ of the spectrum of $M$. This is used to construct manifolds with nontrivial essential spectrum and arbitrarily high multiplicities for an arbitrarily large number of eigenvalues below the essential spectrum.
\end{abstract}

Mathematics Subject Classification (2000). 58J50.

Keywords. Laplace operator, spectrum, Lipschitz convergence, spectral convergence, multiplicities.

\section{Introduction}

In this note we investigate the spectrum of the Laplacian acting on square integrable functions on a complete Riemannian manifold which is not necessarily of finite volume. Our main goal is to understand how this spectrum varies as we vary our manifold continuously with respect to the Lipschitz topology for metric pairs.

Here we mean by a metric pair a pair $(M, A)$ which consists of a metric space $(M, d)$ and a nonempty closed subspace $A \subset M$. For a number $R>0$ denote by $B(A, R)$ the open $R$-neighborhood of $A$ in $M$. The Lipschitz topology for metric pairs is defined as follows (compare [G]).

Definition. A sequence of metric pairs $\left(M_{i}, A_{i}\right)$ converges to the metric pair $(M, A)$ in the Lipschitz topology if there is a sequence of numbers $R_{i} \rightarrow \infty$, a sequence of numbers $\varepsilon_{i} \rightarrow 0$ and for each $i$ a $\left(1+\varepsilon_{i}\right)$-bilipschitz homeomorphism $F_{i}$ of $B\left(A, R_{i}\right) \subset M$ onto a neighborhood of $B\left(A_{i}, R_{i}\right)$ in $M_{i}$ which maps $A$ to $A_{i}$. We call the sequence $\left\{R_{i}\right\}_{i}$ convergence inducing.

\footnotetext{
*Research partially supported by SFB 256 and by SFB 611 .
} 
If the closed sets $A_{i} \subset M_{i}$ and $A \subset M$ consist of single points then we also speak of the Lipschitz topology of pointed metric spaces and Lipschitz convergence of pointed metric spaces (see [G]).

In the sequel we only consider metric pairs $(M, A)$ consisting of a not necessarily connected complete Riemannian manifold $M$ and a closed subset $A$ of $M$ which intersects every connected component of $M$. We call such a pair $(M, A)$ a manifold pair.

For every complete Riemannian manifold $(M, g)$, the spectrum of the Laplacian $\Delta$ acting on square integrable functions is a closed subset $\sigma(M)$ of the half-line $[0, \infty)$. The set $\sigma(M)$ is the disjoint union of the essential spectrum $\sigma_{\mathrm{ess}}(M)$ and the discrete spectrum $\sigma_{\mathrm{disc}}(M)$. The essential spectrum is a closed subset of $\sigma(M)$. The discrete spectrum consists of the eigenvalues of finite multiplicity; they are isolated points in $\sigma(M)$. If $M$ is closed and connected then the essential spectrum of $M$ is empty and $\sigma(M)$ consists of an increasing sequence $0=\lambda_{1}<\lambda_{2}<\cdots$ of nonnegative numbers converging to $\infty$.

If $\left(M_{i}, g_{i}\right)$ are diffeomorphic closed Riemannian manifolds which converge as $i \rightarrow \infty$ in the Lipschitz topology to a closed Riemannian manifold $(M, g)$ then the spectra of $M_{i}$ converge to the spectrum of $M$. However, spectra do not always converge. Namely, consider a sequence $\left(M_{i}, p_{i}\right)_{i}$ of pointed closed connected manifolds which converge in the pointed Lipschitz topology to a complete connected non-compact manifold $(M, p)$ of finite volume.

Let $v \geq 0$ be a lower bound for the essential spectrum of $M$ and assume that $M$ admits at least $k \geq 0$ eigenvalues counted with multiplicities which are smaller than $v$. In [CC1] and [CC2], Colbois and Courtois show that the first $k$ eigenvalues of $M_{i}$ converge to the first $k$ eigenvalues of $M$ if and only if there is a convergence inducing sequence $R_{i} \rightarrow \infty$ and a sequence $r_{i} \rightarrow \infty$ such that for sufficiently large $i$ the smallest Rayleigh quotient of $M_{i}-B\left(p_{i}, R_{i}-r_{i}\right)$ is not smaller than $v$. Recall that the smallest Rayleigh quotient $\mu_{1}(\Omega)$ of an open subset $\Omega$ of a Riemannian manifold $(M, g)$ is defined to be the infimum of all quotients $\mathcal{R}(f)=\int g(d f, d f) / \int f^{2}$ over all nontrivial smooth functions $f$ with compact support in $\Omega$.

We adapt this idea to our more general situation using the following definition.

Definition. Let $\left(M_{i}, A_{i}\right)$ be a sequence of metric pairs converging in the Lipschitz topology to the metric pair $(M, A)$ with a convergence inducing sequence $R_{i} \rightarrow \infty$. A family of open subsets $\Omega_{i} \subset M_{i}-A_{i}$ is called escaping if there is a sequence $r_{i} \rightarrow \infty$ such that $\Omega_{i}$ contains $M_{i}-B\left(A_{i}, R_{i}-r_{i}\right)$.

We use here the notion of Colbois and Courtois in [CC2] even though our definition slightly differs from theirs and our escaping sets do not necessarily "escape" in an intuitive sense.

Denote by $L^{2}(M)$ the Hilbert space of square integrable functions on a Riemannian manifold $M$ and let $H^{1}(M)$ be the Hilbert space of square integrable functions 
on $M$ with square integrable differential. Let $\left(M_{i}, A_{i}\right)$ be a sequence of manifold pairs converging to $(M, A)$ with convergence inducing sequence $\left\{R_{i}\right\}_{i}$ and $\left(1+\varepsilon_{i}\right)$-bilipschitz embeddings $F_{i}$ of $\left(B\left(A, R_{i}\right), A\right)$ into $\left(M_{i}, A_{i}\right)$. We say that a sequence of functions $f_{i} \in L^{2}\left(M_{i}\right)$ converges effectively to a function $f \in L^{2}(M)$ if $\int_{M_{i}-B\left(A_{i}, R_{i}\right)} f_{i}^{2} \rightarrow 0$ and if moreover $\int_{B\left(A, R_{i}\right)}\left(f_{i} \circ F_{i}-f\right)^{2} \rightarrow 0$ as $i \rightarrow \infty$. We show

Theorem A. Let $\left(M_{i}, A_{i}\right)$ be a sequence of manifold pairs which converges in the Lipschitz topology to the manifold pair $(M, A)$. Let $\Omega_{i} \subset M_{i}$ be an escaping family of sets and let $v \leq \liminf _{i \rightarrow \infty} \mu_{1}\left(\Omega_{i}\right)$. Then the sets $\sigma\left(M_{i}\right) \cap[0, v)$ converge as $i \rightarrow \infty$ in the Hausdorff topology for closed subsets of $[0, v)$ to $\sigma(M) \cap[0, v)$. Moreover, every function $f \in H^{1}(M)$ whose spectral measure is supported in $[0, v)$ is an effective limit of functions $f_{i} \in H^{1}\left(M_{i}\right)$ whose spectral measures converge weakly to the spectral measure of $f$.

For closed pointed Riemannian manifolds $\left(M_{i}, p_{i}\right)$ which converge to a complete manifold $(M, p)$ of finite volume we can combine our Theorem A with standard compactness results for solutions of elliptic equations to conclude that up to passing to a subsequence, eigenfunctions on $M_{i}$ for small eigenvalues converge to eigenfunctions on $M$.

One can also ask about convergence properties for sequences of eigenfunctions on our manifolds $M_{i}$ for eigenvalues which are uniformly bounded but bigger than $v=\liminf _{i \rightarrow \infty} \mu_{1}\left(\Omega_{i}\right)$ for every escaping family of sets $\Omega_{i} \subset M_{i}$. By the results of Colbois and Courtois, such functions might not be visible in the spectrum of the limit manifold $M$. In some special cases, suitable renormalizations of these functions viewed as functions on larger and larger subsets of $M$ converge up to passing to a subsequence locally uniformly to an eigenfunction on $M$ which however is in general not square integrable. In Section 3 we look at a rather special class of examples where such a convergence can be deduced.

Namely, let $N$ be a closed two-sided hypersurface in a closed manifold $M$. Then $N$ has a tubular neighborhood $U$ which is diffeomorphic to $N \times(-1,1)$. We consider a family $g_{s}$ of Riemannian metrics on $M$ which depend smoothly on $s \in(0,1]$ and which are warped product metrics on $U=N \times(-1,1)$. More precisely, we assume that there is a smooth family $h_{s}(s \in[0,1])$ of smooth Riemannian metrics on $N$ and a smooth function $\rho:(0,1] \times[-1,1] \rightarrow(0, \infty)$ such that the restriction of $g_{s}$ to $N \times(-1,1)$ is of the form $g_{s}=\frac{1}{s^{2}+t^{2}} d t^{2}+\rho(s, t) h_{s}(s \in(0,1])$. As $s \searrow 0$ these metrics converge uniformly on compact subsets of $N \times([-1,0) \cup(0,1])$ to a complete metric $g_{0}$. We assume that the metrics $g_{s}$ can be extended to smooth Riemannian metrics on $M-U$ which depend smoothly on $s \in[0,1]$. We assume moreover that $\rho(s, t) \searrow 0$ as $(s, t) \rightarrow(0,0)$.

The following observation extends a result of Judge $[\mathrm{J}]$ with a similar but somewhat shorter proof. For its formulation, we mean now by an eigenfunction a solution of 
the differential equation $\Delta-\lambda=0$ for some $\lambda \in \mathbb{R}$ which is not necessarily square integrable.

Theorem B. Let $s_{i} \subset(0,1]$ be a sequence converging to 0 and let $f_{i}$ be an eigenfunction on $\left(M, g_{s_{i}}\right)$ with respect to an eigenvalue $\lambda_{i}$. If the sequence $\lambda_{i}$ converges to some $\lambda \geq 0$ then after passing to a subsequence and possibly a renormalization the functions $f_{i}$ converge uniformly on compact subsets of $M-N$ to a nonzero eigenfunction for $g_{0}$ with respect to the eigenvalue $\lambda$.

We also give an example which illustrates that the limit function is in general not square integrable, even if the curvatures and the volumes of all the metrics $g_{s}$ are uniformly bounded.

In Section 4 we construct manifolds of bounded nonpositive sectional curvature and with controlled spectral properties.

Theorem C. For every $n \geq 2, k>0, m>0$ there is a smooth Riemannian manifold $M$ of dimension $n$ and curvature contained in $[-1,0]$ and with the following additional properties.

(1) The essential spectrum $\sigma_{\mathrm{ess}}(M)$ of $M$ is not empty and $M$ has infinitely many eigenvalues below $\sigma_{\mathrm{ess}}(M)$.

(2) For $2 \leq j \leq k$ the multiplicity of the $j$-th eigenvalue of the Laplacian is at least $m$.

In the case $n=2$ we can choose $M$ to have constant curvature -1 .

Our construction can also be used to obtain for any $n \geq 2$ and for given $k>0$, $m>0$ a compact $n$-dimensional manifold of nonpositive curvature with the property that for $2 \leq j \leq k$ the multiplicity of the $j$-th eigenvalue is at least $k$. However, in this case a much stronger result is due to Colin de Verdière [CV2]. He showed that for every closed manifold $M$ of dimension at least 3 and an arbitrary finite sequence of nonnegative numbers of the form $0=\lambda_{0}<\lambda_{1} \leq \cdots \leq \lambda_{m}(m \geq 0)$ there is a Riemannian metric on $M$ whose $i$-th eigenvalue $(0 \leq i \leq m)$ is just $\lambda_{i}$.

\section{Proof of Theorem A}

This section is devoted to the proof of Theorem A. We continue to use the assumptions and notations from the introduction. In particular, we denote by $(M, g)$ a complete Riemannian manifold and by $A$ a nonempty closed subset of $M$.

For functions $f, h$ on $(M, g)$ denote by $(f, h)_{2}=\int_{M} f h$ their $L^{2}$-inner product and let $(\nabla f, \nabla h)_{2}=\int_{M} g(\nabla f, \nabla h)$ be the $L^{2}$-inner product of their gradients. Write 
also $\|f\|=\sqrt{(f, f)_{2}}$ and $\|\nabla f\|=\sqrt{(\nabla f, \nabla f)_{2}}$. We denote by $H^{1}(M)$ the Hilbert space of square integrable functions on $M$ with square integrable differential with the inner product $(f, h)_{2}+(\nabla f, \nabla h)_{2}$. For every nonzero function $f \in H^{1}(M)$ the Rayleigh quotient of $f$ is defined by $\mathcal{R}(f)=\|\nabla f\|^{2} /\|f\|^{2}$.

We begin our argument with a general estimate of Rayleigh quotients for suitably chosen functions on $M$.

Lemma 2.1. For $\varepsilon>0$ there is a number $\delta=\delta(\varepsilon)>0$ with the following property. Let $M$ be a complete Riemannian manifold, let $U, V$ be open subsets of $M$ with disjoint closures and let $u, v$ be smooth functions on $M$ with compact supports in $U, V$. If $\mathcal{R}(u+v)<\mu_{1}(V)-\varepsilon$ and $|\mathcal{R}(u+v)-\mathcal{R}(u)|<\delta$ then $\|v\|^{2}<\varepsilon\|u\|^{2}$ and $\|\nabla v\|^{2}<\varepsilon\left(\|\nabla u\|^{2}+2 \varepsilon\|u\|^{2}\right)$.

Proof. Let $u, v$ be as in the lemma. Since $v$ is supported in $V$ we have $\mathcal{R}(v) \geq$ $\mu_{1}(V)>\mathcal{R}(u+v)+\varepsilon$.

Write

$$
a=\|\nabla u\|^{2}, \quad b=\|u\|^{2}, \quad c=\|\nabla v\|^{2}, \quad d=\|v\|^{2} .
$$

Since the supports of $u$ and $v$ are disjoint we have $\|u+v\|^{2}=b+d$ and $\|\nabla(u+v)\|^{2}=$ $a+c$ and consequently $\frac{c}{d}=\mathcal{R}(v)>\mathcal{R}(u+v)+\varepsilon=\frac{a+c}{b+d}+\varepsilon$. This implies that

$$
\varepsilon \frac{\|v\|^{2}}{\|u\|^{2}}=\varepsilon \frac{d}{b}<\frac{a+c}{b+d}-\frac{a}{b}=\mathcal{R}(u+v)-\mathcal{R}(u) .
$$

Thus if $|\mathcal{R}(u)-\mathcal{R}(u+v)|<\delta(\varepsilon)=\varepsilon^{2}$ then our above inequality shows that $\|v\|^{2}<\varepsilon\|u\|^{2}$.

Using again that the supports of $u$ and $v$ are disjoint we obtain from this that

$$
\mathcal{R}(u)+\varepsilon^{2}>\mathcal{R}(u+v)>\frac{\|\nabla u\|^{2}+\|\nabla v\|^{2}}{(1+\varepsilon)\|u\|^{2}}=\frac{\mathcal{R}(u)}{1+\varepsilon}+\frac{\|\nabla v\|^{2}}{(1+\varepsilon)\|u\|^{2}}
$$

and therefore

$$
\varepsilon \mathcal{R}(u)+\varepsilon^{2}(1+\varepsilon)>\frac{\|\nabla v\|^{2}}{\|u\|^{2}}
$$

and $\|\nabla v\|^{2}<\varepsilon\|\nabla u\|^{2}+\varepsilon^{2}(1+\varepsilon)\|u\|^{2}$. This shows the lemma.

For a closed subset $A$ of a complete Riemannian manifold $M$ and a number $r>0$ let as before $B(A, r)$ be the open $r$-neighborhood of $A$ in $M$. In the sequel we always assume without further mentioning that the boundaries of our sets $B(A, r)$ are smooth. This can be achieved with a small deformation of $B(A, r)$ near its boundary. We also write $M=B(A, \infty)$.

The next lemma is a technical tool which allows us to find for every function $f \in H^{1}(M)$ with controlled Rayleigh quotient a function $f^{\prime} \in H^{1}(M)$ which is 
close to $f$ and to which Lemma 2.1 can be applied. In a less explicit form, this lemma was used by Colbois and Courtois [CC2].

Lemma 2.2. For $C>0, \rho>0$ there is a number $R_{0}=R_{0}(C, \rho)>0$ as follows. If $f \in H^{1}(M)$ is such that $\|f\|^{2}=1$ and $\mathcal{R}(f)<C$ then there is a function $u=u(f)$ with the following properties.

(1) $u$ has values in $[0,1]$ and $|\nabla u| \leq 1$ pointwise.

(2) $u=u_{1}+u_{2}$ where $u_{1}$ is supported in $B\left(A, R_{0}\right)$ and the support of $u_{2}$ is disjoint from the support of $u_{1}$ and contained in $M-A$.

$$
\int_{M}\left((f-f u)^{2}+\|\nabla(f-f u)\|^{2}\right)<\rho .
$$

Proof. Using the notations from the lemma, choose a number $k>0$ such that $k \rho / 4>$ $1+C$. Notice that $k$ only depends on $\rho, C$. For $m \leq k$ define $E_{m}=\{x \mid \operatorname{dist}(x, A) \in$ $[6 m, 6 m+6)\}$. Then $B(A, 6 k+6)-B(A, 6)$ is the disjoint union of the $k$ spherical shells $E_{m}$.

Let $f \in H^{1}(M)$ be such that $\|f\|^{2}=1$ and $\|\nabla f\|^{2}<C$. Then $\int\|\nabla f\|^{2}+f^{2}<$ $C+1$ and therefore, by our choice of $k$, there is some $m \in\{1, \ldots, k\}$ such that

$$
\int_{E_{m}}\|\nabla f\|^{2}+f^{2}<\rho / 4
$$

For this number $m \leq k$, choose a smooth function $\tilde{u}_{1}: \mathbb{R} \rightarrow[0,1]$ which is supported in $(-\infty, 6 m+2)$, equals 1 on $(-\infty, 6 m]$ and whose gradient is pointwise bounded in norm by 1 . Similarly, let $\tilde{u}_{2}: \mathbb{R} \rightarrow[0,1]$ be a smooth function which is supported in $(6 m+4, \infty)$, equals 1 on $[6 m+6, \infty)$ and whose gradient is pointwise bounded in norm by 1 . Define $u_{i}=\tilde{u}_{i}(\operatorname{dist}(A, \cdot))$ and $u=u_{1}+u_{2}$. For $R_{0}=6 k+6$ the function $u_{1}$ is supported in $B\left(A, R_{0}\right)$. The support of $u_{2}$ is contained in $M-A$ and it is disjoint from the support of $u_{1}$.

The function $1-u$ is supported in the shell $E_{m}$ and it satisfies $|1-u| \leq 1$, $\|\nabla(1-u)\| \leq 1$ pointwise. Therefore we have

$$
\int_{M}(f-f u)^{2}=\int_{E_{m}} f^{2}(1-u)^{2} \leq \int_{E_{m}} f^{2}<\rho / 4
$$

and

$$
\begin{aligned}
\int_{M}\|\nabla(f-f u)\|^{2} & =\int_{E_{m}}\|(1-u) \nabla f+f \nabla(1-u)\|^{2} \\
& \leq \int_{E_{m}}\|\nabla f\|^{2}+2(1-u) f g(\nabla f, \nabla(1-u))+f^{2} \\
& <\rho / 4+2 \int_{E_{m}} f\|\nabla f\| \leq 3 \rho / 4 .
\end{aligned}
$$


In other words, our function $u$ has the required properties.

For an open subset $\Omega$ of $M$ with smooth boundary we denote by $H^{1}(\Omega)$ the closure in $H^{1}(M)$ of the space of smooth functions with compact support in $\Omega$. Then $H^{1}(\Omega)$ is a closed linear subspace of $H^{1}(M)$.

The self-adjoint extension of the Laplacian $\Delta_{\Omega}$ on $\Omega$ with Dirichlet boundary conditions is the self-adjoint operator of the quadratic form $(f, u) \rightarrow(\nabla f, \nabla u)_{2}$. The domain of $\Delta_{\Omega}^{1 / 2}$ is the Hilbert space $H^{1}(\Omega)$. We denote by $\sigma(\Omega) \subset[0, \infty)$ the spectrum of $\Delta_{\Omega}$.

The next lemma is the key technical result needed for the proof of Theorem A.

Lemma 2.3. For $\varepsilon>0, C>0, \chi \in(0, \varepsilon / 2)$ there is a number $R=R(\varepsilon, C, \chi)>0$ and a number $v=v(\varepsilon, C, \chi)>0$ such that the following is satisfied. Let $M$ be a complete Riemannian manifold and let $A \subset M$ be a closed set. Then there is a continuous linear map $L: H^{1}(M) \rightarrow H^{1}(M)$ with the following properties.

(1) The range of $L$ is contained in $H^{1}(B(A, R))$.

(2) $L$ extends continuously to $L^{2}(M)$, and $(L \alpha, \beta)_{2}=(\alpha, L \beta)_{2}$ for all $\alpha, \beta \in$ $L^{2}(M)$.

(3) If $\lambda<\min \left\{\mu_{1}(M-A)-\varepsilon, C\right\}, r \in[R, \infty]$ and if the spectral measure of $f \in H^{1}(B(A, r))$ is contained in $[\lambda-v, \lambda+v]$ then $\|f-L f\|^{2} \leq \chi\|f\|^{2}$ and $\|\nabla(L f)-\nabla f\|^{2}<\chi\|\nabla f\|^{2}$.

Proof. Let $M$ be a complete Riemannian manifold and let $A \subset M$ be a closed set. The proof of our lemma is divided into three steps.

Step 1 . We claim that for every $\delta \in(0,1), C>0$ there is a number $\beta=\beta(\delta, C)>0$ with the following property. Let $f \in H^{1}(M)$ be a normalized function with $\mathcal{R}(f)<$ $\min \left\{\mu_{1}(M-A), C\right\}-\delta$. Let $\rho \in\left(0, \frac{\delta}{2}\right)$ be an arbitrary number which is small enough that $\frac{C+\rho}{1-\rho}-C<\delta / 2$ and let $u=u_{1}+u_{2}$ be the function constructed in Lemma 2.2 for $f$ and the constants $C, \rho / 2>0$; then $\int\left(f u_{1}\right)^{2} \geq \beta$.

Namely, by Lemma 2.2 we have $\left|\|f\|^{2}-\|f u\|^{2}\right|+\left|\|\nabla f\|^{2}-\|\nabla(f u)\|^{2}\right|<\rho$ and therefore since $f$ is normalized and $u \leq 1$ we obtain that

$$
\mathcal{R}(f)-\rho=\frac{\|\nabla f\|^{2}-\rho}{\|f\|^{2}} \leq \mathcal{R}(u f) \leq \frac{\|\nabla f\|^{2}+\rho}{\|f\|^{2}-\rho} .
$$

By our choice of $\rho$ and the fact that $\mathcal{R}(f)<C$ we conclude that $|\mathcal{R}(u f)-\mathcal{R}(f)|<$ $\delta / 2$ and hence $\mathcal{R}(u f)<\min \left\{\mu_{1}(M-A), C\right\}-\delta / 2$. Now

$$
\mathcal{R}(u f)=\frac{\left\|\nabla\left(u_{1} f\right)\right\|^{2}+\left\|\nabla\left(u_{2} f\right)\right\|^{2}}{\left\|u_{1} f\right\|^{2}+\left\|u_{2} f\right\|^{2}}
$$


and consequently since $u_{2} f$ is supported in $M-A$ we obtain that

$$
\begin{aligned}
\min \left\{\mu_{1}(M-A), C\right\}-\delta / 2 & >\frac{\left\|\nabla\left(u_{1} f\right)\right\|^{2}+\mu_{1}(M-A)\left\|u_{2} f\right\|^{2}}{\left\|u_{1} f\right\|^{2}+\left\|u_{2} f\right\|^{2}} \\
& \geq \frac{\mu_{1}(M-A)\left\|u_{2} f\right\|^{2}}{\left\|u_{1} f\right\|^{2}+\left\|u_{2} f\right\|^{2}}
\end{aligned}
$$

and hence

$$
\left\|u_{1} f\right\|^{2} \geq \delta\left\|u_{2} f\right\|^{2} / 2 C
$$

The existence of a constant $\beta=\beta(\delta, C)$ as stated above now follows from the fact that $\left\|u_{1} f\right\|^{2}+\left\|u_{2} f\right\|^{2} \geq 1-\rho \geq 1-\frac{\delta}{2}$ by Lemma 2.2.

Step 2. Let $\varepsilon>0$ and let $\chi<\varepsilon / 2$. Let $C \geq 1$, let $\delta=\delta(\chi / 2 C)<\chi / 2$ be as in Lemma 2.1 and let $\beta=\beta(\delta, C) \leq 1$ be the constant from Step 1 above. Notice that $\beta$ only depends on $\varepsilon, \chi, C$. Choose $\rho \in(0, \min \{\chi / 2, \delta \beta / 4(3+C)\})$ small enough that $\frac{C+\rho}{1-\rho}-C<\delta / 4$. Let $R_{0}=R_{0}(C, \rho)$ be the constant from Lemma 2.2 for $\rho$; notice that $R_{0}$ only depends on $\varepsilon, \chi, C$. Let $r \in\left[R_{0}+2, \infty\right]$ and for simplicity write $\Omega=B(A, r)$.

We use the spectral theorem in the following form (see [D]). There is a finite measure $\mu$ on $\sigma(\Omega) \times \mathbb{N}$ and a unitary operator $U: L^{2}(\Omega) \rightarrow L^{2}(\sigma(\Omega) \times \mathbb{N}, d \mu)$ as follows. Define $h(s, n)=s$; then $f \in L^{2}(\Omega)$ is contained in the domain of $\Delta_{\Omega}$ if and only if $h U(f) \in L^{2}(\sigma(\Omega) \times \mathbb{N}, d \mu)$, and if this is the case we have $U \Delta_{\Omega} U^{-1}(U f)=h U(f)$. The spectral measure of such a function $f$ is supported in an interval $[\lambda-\kappa, \lambda+\kappa]$ if and only if the function $U f$ is supported in $[\lambda-\kappa, \lambda+\kappa] \times \mathbb{N}$. Since $(u, q) \rightarrow(\nabla u, \nabla q)_{2}$ is the quadratic form of $\Delta_{\Omega}^{1 / 2}$ this implies that for every $q \in H^{1}(\Omega)$ we have

$$
\begin{aligned}
\left|(\nabla f, \nabla q)_{2}-\lambda(f, q)_{2}\right| & =\left|\int h(U f)(U q) d \mu-\lambda \int(U f)(U q) d \mu\right| \\
& \leq \kappa\left|\int(U f)(U q) d \mu\right|=\kappa(f, q)_{2} .
\end{aligned}
$$

Using this inequality for $u=f$ we obtain in particular that the Rayleigh quotient of $f$ is contained in the interval $[\lambda-\kappa, \lambda+\kappa]$. Moreover, if $f$ and $q$ are contained in the domain of $\Delta_{\Omega}$ and if their spectral measures are supported on disjoint subsets of $\sigma(\Omega)$ then we have $(f, q)_{2}=(\nabla f, \nabla q)_{2}=0$.

Let $\lambda<\min \left\{\mu_{1}(M-A), C\right\}-\varepsilon$ and let $f \in H^{1}(\Omega)$ be a normalized function with spectral measure contained in $[\lambda-\delta \sqrt{\beta} / 4, \lambda+\delta \sqrt{\beta} / 4]$. Then the Rayleigh quotient of $f$ is not bigger than $\lambda+\delta / 4<\min \left\{\mu_{1}(M-A), C\right\}-3 \delta / 4$. Let $u=u_{1}+u_{2}$ be the function for $f$ as in Lemma 2.2; then as in Step 1 above we obtain that

$$
\frac{\|\nabla f\|^{2}-\rho}{\|f\|^{2}} \leq \mathcal{R}(u f) \leq \frac{\|\nabla f\|^{2}+\rho}{\|f\|^{2}-\rho}
$$


and therefore by our choice of $\rho$ we have $|\mathcal{R}(u f)-\mathcal{R}(f)|<\delta / 4$ and, in particular, $\mathcal{R}(u f) \in[\lambda-\delta / 2, \lambda+\delta / 2] \subset\left(0, \mu_{1}(M-A)-\varepsilon / 2\right]$.

On the other hand, from the properties of the spectral measure for $f$ and the fact that $f$ is normalized we infer that

$$
\left|\int g(\nabla f, \nabla \psi)-\lambda \int \psi f\right| \leq \delta \sqrt{\beta}\|\psi\| / 4
$$

for every smooth function $\psi$ on $\Omega$ with compact support. For $\psi=u_{1} f \in H^{1}(\Omega)$ and with the notation from the proof of Lemma 2.2 above this means that

$$
\left|\int\left\|\nabla u_{1} f\right\|^{2}+\int_{E_{m}} g\left(\nabla\left(f\left(1-u_{1}\right)\right), \nabla\left(u_{1} f\right)\right)-\lambda \int u_{1} f^{2}\right| \leq \delta \sqrt{\beta}\left\|u_{1} f\right\| / 4 .
$$

Moreover we have $\left\|u_{1} f\right\| \geq \sqrt{\beta}$ by the choice of $\beta$ and Step 1 .

Now the intersection of the supports of $u_{1}$ and $1-u_{1}$ is contained in $E_{m}$ and consequently $\left|\int g\left(\nabla\left(f\left(1-u_{1}\right)\right), \nabla\left(u_{1} f\right)\right)\right| \leq \int_{E_{m}}\|\nabla f\|^{2}+f^{2}+2 f\|\nabla f\| \leq 3 \rho$ and hence we conclude as in Step 1 that

$$
\begin{aligned}
\left|\int\left\|\nabla u_{1} f\right\|^{2}-\lambda \int\left(u_{1} f\right)^{2}\right| & \leq 3 \rho+\lambda \int_{E_{m}}\left(1-u_{1}\right) u_{1} f^{2}+\delta \sqrt{\beta}\left\|u_{1} f\right\| / 4 \\
& \leq(3+\lambda) \rho+\delta \sqrt{\beta}\left\|u_{1} f\right\| / 4 \\
& \leq(3+\lambda) \rho+\delta\left\|u_{1} f\right\|^{2} / 4 \leq \delta \int\left(u_{1} f\right)^{2} / 2 .
\end{aligned}
$$

For the last of these inequalities, recall that $\lambda<C, \rho<\delta \beta / 4(3+C)$ and hence $(3+\lambda) \rho<(3+C) \rho<\delta \beta / 4 \leq \delta \int\left(u_{1} f\right)^{2} / 4$ by the choice of $\beta$.

In particular, the Rayleigh quotient $\mathcal{R}\left(u_{1} f\right)$ is contained in $[\lambda-\delta / 2, \lambda+\delta / 2]$ and $\left|\mathcal{R}\left(u_{1} f\right)-\mathcal{R}(u f)\right|<\delta$.

Now we can apply Lemma 2.1 to the functions $u_{1} f$ and $u_{2} f$ and deduce that

$$
\left\|u f-u_{1} f\right\|^{2}<\chi\left\|u_{1} f\right\|^{2} / 2 C<\chi / 2
$$

and

$$
\left\|\nabla\left(u f-u_{1} f\right)\right\|^{2}<\chi\left\|\nabla u_{1} f\right\|^{2} / 2 C<\chi / 2
$$

and therefore also $\left\|u_{1} f-f\right\|^{2}<\chi$ and $\left\|\nabla\left(u_{1} f-f\right)\right\|^{2}<\chi$. As a consequence, we have $\int_{M-B\left(A, R_{0}\right)} f^{2}<\chi$ and $\int_{M-B\left(A, R_{0}\right)}\|\nabla f\|^{2}<\chi$.

Step 3. Let $v: M \rightarrow[0,1]$ be a smooth function with support in $B\left(A, R_{0}+2\right)$ and which is constant 1 on $B\left(A, R_{0}\right)$. We may choose our function in such a way that its gradient $\nabla v$ is pointwise bounded in norm by 1 . For a function $f \in H^{1}(M)$ define 
$L f=v f$. Then $L: H^{1}(M) \rightarrow H^{1}(M)$ is clearly linear, extends continuously to $L^{2}(M)$ and satisfies $(L \alpha, \beta)_{2}=(\alpha, L \beta)_{2}$ for all $\alpha, \beta \in L^{2}(M)$. Since $|v| \leq 1$ and $\|\nabla v\| \leq 1$ pointwise the map $L$ is continuous. More precisely, we have $\|L f-f\|^{2} \leq$ $\int_{M-B\left(A, R_{0}\right)} f^{2}$ and

$$
\|\nabla L f-\nabla f\|^{2} \leq \int_{B\left(A, R_{0}+2\right)-B\left(A, R_{0}\right)} f^{2}+\int_{M-B\left(A, R_{0}\right)}\|\nabla f\|^{2} .
$$

This together with Step 2 above shows the second and the third part of our lemma.

We are left with showing that the image of $H^{1}(M)$ under the map $L$ is contained in $H^{1}\left(B\left(A, R_{0}+2\right)\right)$. For this observe that for every smooth function $f$ on $M$ with compact support the function $L f$ is smooth and compactly supported in $B\left(A, R_{0}+2\right)$. Since compactly supported smooth functions are dense in $H^{1}(M)$ and since $L$ is continuous, functions with compact support in $B\left(A, R_{0}+2\right)$ are dense in the range of $L$. This shows the lemma.

Corollary 2.4. For $\varepsilon>0, C>0$ and $\delta<\varepsilon / 2$ there are numbers $\rho=\rho(\varepsilon, C, \delta)>0$ and $\kappa=\kappa(\varepsilon, C, \delta)<\delta / 2$ such that for every complete Riemannian manifold $M$ and every closed subset $A \subset M$ the following holds.

(1) Let $\lambda \in\left[0, \min \left\{\mu_{1}(M-A), C\right\}-\varepsilon\right] \cap \sigma(M)$ and let $f \in H^{1}(M)$ be a function whose spectral measure is supported in $[\lambda-\kappa, \lambda+\kappa]$. Then there is a function $\tilde{f} \in H^{1}(B(A, \rho))$ with spectral measure supported in $[\lambda-\delta, \lambda+\delta]$ and such that $\|f-\tilde{f}\|^{2}<\delta\|f\|^{2}$.

(2) Let $\lambda \in\left[0, \min \left\{\mu_{1}(M-A), C\right\}-\varepsilon\right] \cap \sigma(B(A, \rho))$ and let $f \in H^{1}(B(A, \rho))$ be a function whose spectral measure is supported in $[\lambda-\kappa, \lambda+\kappa]$. Then there is a function $\tilde{f} \in H^{1}(M)$ with spectral measure supported in $[\lambda-\delta, \lambda+\delta]$ and such that $\|f-\tilde{f}\|^{2}<\delta\|f\|^{2}$.

Proof. Let $\varepsilon \in(0,1], \delta<\varepsilon / 2$ and let $C \geq 1$. Define $\kappa=\delta^{3} /(C+1)$ and let $\rho=R\left(\varepsilon / 2, C, \kappa^{2}\right)$ be as in Lemma 2.3. Denote by $L: H^{1}(M) \rightarrow H^{1}(B(A, \rho))$ the linear map from Lemma 2.3.

Let $v=v\left(\varepsilon / 2, C, \kappa^{2}\right)<\kappa / 2$ be as in Lemma 2.3 and let $\lambda \in \sigma(M) \cap$ $\left[0, \min \left\{\mu_{1}(M-A), C\right\}-\varepsilon\right]$. Let $f$ be a normalized function on $M$ with spectral measure supported in $[\lambda-v, \lambda+v]$. Then the Rayleigh quotient of $f$ is not bigger than $\lambda+v<\min \left\{\mu_{1}(M-A), C\right\}-\varepsilon / 2$. Moreover, since $f$ is normalized we obtain that

$$
\left|\int g(\nabla f, \nabla u)-\lambda \int u f\right| \leq 2 v\|u\|
$$

for every smooth function $u$ on $M$ with compact support.

By construction of the operator $L$, the function $L f$ lies in the domain of $\Delta_{B(A, \rho)}$. Moreover by Lemma 2.3 we have $\|L f-f\|^{2}<\kappa^{2}\|f\|^{2}$ and $\|\nabla(L f-f)\|^{2}<$ 
$\kappa^{2}\|\nabla f\|^{2}$. Using the spectral theorem for the operator $\Delta_{B(A, \rho)}$ acting on $L^{2}(B(A, \rho))$ with Dirichlet boundary conditions we obtain that the function $L f$ admits an $L^{2}$ orthogonal decomposition $L f=\alpha+\varphi+\beta$ where the spectral measure of $\alpha$ is supported in $\left[0, \lambda-\delta^{2}\right]$, the spectral measure of $\beta$ is supported in $[\lambda+\delta, \infty)$ and the spectral measure of $\varphi$ is supported in $\left[\lambda-\delta^{2}, \lambda+\delta\right]$. Since $\|L f-f\|^{2}<\kappa^{2}\|f\|^{2}$ by construction, for the first part of our lemma it is enough to show that the square norms of $\alpha$ and $\beta$ are bounded from above by a fixed multiple of $\delta$.

For an estimate of $\|\alpha\|^{2}$, observe that

$$
\|\alpha\|^{2}=\int \alpha(L f)=\int \alpha f+\int \alpha(L f-f) \leq \int \alpha f+\kappa\|\alpha\|
$$

since $f$ is normalized by assumption and therefore using the fact that $\mathcal{R}(f)=$ $\|\nabla f\|^{2}<C$ we obtain

$$
\begin{aligned}
\left(\lambda-\delta^{2}\right)\|\alpha\|^{2} & \geq\|\nabla \alpha\|^{2}=\int g(\nabla \alpha, \nabla(L f)) \geq \int g(\nabla \alpha, \nabla f)-\kappa\|\nabla \alpha\|\|\nabla f\| \\
& \geq \lambda \int \alpha f-\kappa(\|\alpha\|+\sqrt{C}\|\nabla \alpha\|) \geq \lambda\|\alpha\|^{2}-\kappa\|\alpha\|(\lambda+2+C) .
\end{aligned}
$$

This shows that $\|\alpha\| \leq 2 \kappa(C+1) / \delta^{2}<\delta$ by our choice of $\kappa$ and the fact that $\lambda \leq C$.

On the other hand, the square norm of $\beta$ can be estimated as follows. By construction and Lemma 2.3 we have

$$
\begin{aligned}
\left(1+\kappa^{2}\right)(\lambda+\kappa) & \geq\left(1+\kappa^{2}\right)\|\nabla f\|^{2} \geq\|\nabla(L f)\|^{2} \\
& =\|\nabla \alpha\|^{2}+\|\nabla \varphi\|^{2}+\|\nabla \beta\|^{2} \\
& \geq\left(\lambda-\delta^{2}\right)\|\varphi\|^{2}+(\lambda+\delta)\|\beta\|^{2} .
\end{aligned}
$$

Since $\|\varphi\|^{2}+\|\beta\|^{2}=\|L f\|^{2}-\|\alpha\|^{2} \geq 1-\kappa^{2}-\|\alpha\|^{2} \geq 1-2 \delta^{2}$ we obtain from this that

$$
\left(1+\kappa^{2}\right)(\lambda+\kappa) \geq\left(1-2 \delta^{2}\right)\left(\lambda-\delta^{2}\right)+\delta\|\beta\|^{2}
$$

and hence $\delta\|\beta\|^{2} \leq \kappa+\kappa^{2}(\lambda+\kappa)+\delta^{2}+2 \delta^{2}\left(\lambda-\delta^{2}\right)$ and $\|\beta\|^{2} \leq \delta(3+2 \lambda)$. This estimate concludes the first part of our corollary.

To show the second part of the corollary, notice that we may always increase $\rho$ without changing our estimates and therefore we may assume that the first part of our corollary is valid for $\rho$ and the constants $\varepsilon>0, C>0, \delta^{2}>0$. Let $\kappa=$ $\kappa\left(\varepsilon, C, \delta^{2}\right)<\delta^{4} / 8$ be the constant from the first part of our corollary. Let $\lambda \in$ $\sigma(B(A, \rho)) \cap\left[0, \min \left\{\mu_{1}(M-A), C\right\}-\varepsilon\right]$ and let $f \in H^{1}(B(A, \rho))$ be a normalized function with spectral measure supported in $[\lambda-\kappa, \lambda+\kappa]$. Then $f$ as a function from $H^{1}(M)$ admits an orthogonal decomposition $f=\alpha+\varphi+\beta$ such that the spectral measure of $\alpha$ is supported in $\left[0, \lambda-2 \delta^{2}\right]$, the spectral measure of $\varphi$ is supported in 
$\left[\lambda-2 \delta^{2}, \lambda+\delta\right]$ and the spectral measure of $\beta$ is supported in $[\lambda+\delta, \infty)$. As above it is now enough to control the square norms of $\alpha$ and $\beta$.

For this we use our above strategy and show first that $\|\beta\|^{2} \leq 3 \delta+\|\alpha\|^{2}\left(\lambda-2 \delta^{2}\right) / \delta$. Namely, notice that the functions $\alpha, \varphi, \beta$ are $L^{2}$-orthogonal and also orthogonal with respect to the inner product of $H^{1}(M)$. Thus the Rayleigh quotient $\mathcal{R}(f)$ of our function $f$ can be estimated as

$$
\lambda+\kappa \geq \mathcal{R}(f)=\|\nabla \alpha\|^{2}+\|\nabla \varphi\|^{2}+\|\nabla \beta\|^{2} \geq\left(\lambda-2 \delta^{2}\right)\|\varphi\|^{2}+(\lambda+\delta)\|\beta\|^{2} .
$$

Since $1-\|\alpha\|^{2}=\|\varphi\|^{2}+\|\beta\|^{2}$ we obtain from this that

$$
\lambda+\kappa \geq\left(1-\|\alpha\|^{2}\right)\left(\lambda-2 \delta^{2}\right)+\delta\|\beta\|^{2}
$$

and hence $\delta\|\beta\|^{2} \leq \kappa+2 \delta^{2}+\left(\lambda-2 \delta^{2}\right)\|\alpha\|^{2}$ from which our above claim is immediate (recall that $\kappa \leq \delta^{4}$ by assumption).

We are left with estimating $\|\alpha\|^{2}$. For this let $L: H^{1}(M) \rightarrow H^{1}(B(A, \rho))$ be the operator as in Lemma 2.3. Since the spectral measure for $f$ as a function on $B(A, \rho)$ is contained in $[\lambda-\kappa, \lambda+\kappa]$ we deduce from Lemma 2.3 that $\|L f-f\|^{2}<\delta^{2}$.

The function $\alpha$ can be decomposed into a finite orthogonal sum of functions with spectral measure supported in a subinterval of $\left[0, \lambda-2 \delta^{2}\right]$ of length smaller than $\kappa$. We apply the first part of our corollary to these functions and obtain a decomposition $L \alpha=\zeta_{1}+\zeta_{2}$ where the spectral measure of $\zeta_{1}$ is supported in $\left[0, \lambda-\delta^{2}\right]$ and we have $\left\|\zeta_{2}\right\|^{2}=\left\|L \alpha-\zeta_{1}\right\|^{2}<\delta^{2}\|\alpha\|^{2}$. However the spectral measure of $f$ as a function in $H^{1}(B(A, \rho))$ is supported in $[\lambda-\kappa, \lambda+\kappa]$ and therefore $\zeta_{1}$ is orthogonal to $f$. Thus $(L \alpha, f)_{2}=\left(\zeta_{2}, f\right) \leq \delta\|\alpha\|$. On the other hand, $(L \alpha, f)_{2}=(\alpha, L f)_{2}=$ $(\alpha, f)_{2}+(\alpha, L f-f)_{2} \geq\|\alpha\|^{2}-\delta\|\alpha\|$. Together with the above this shows that $2 \delta\|\alpha\| \geq\|\alpha\|^{2}$ which is only possible if $\|\alpha\|^{2} \leq 4 \delta^{2}$. Then $\|\beta\|^{2} \leq \delta(4+\lambda)$ which finishes the proof of the corollary.

Now we are ready to show the main result of this section.

Proposition 2.5. Let $\left(M_{i}, A_{i}\right)$ be a sequence of manifold pairs which converges in the Lipschitz topology to the manifold pair $(M, A)$ with convergence inducing sequence $R_{i} \rightarrow \infty$. Assume that there is an escaping family of sets $\Omega_{i} \subset M_{i}$ such that $\liminf _{i \rightarrow \infty} \mu_{1}\left(\Omega_{i}\right) \geq c>0$. Let $\sigma\left(M_{i}\right) \subset[0, \infty)$ be the spectrum of $M_{i}$ and let $\sigma(M)$ be the spectrum of $M$. Then the following is satisfied.

(1) The sets $\sigma\left(M_{i}\right) \cap[0, c)$ converge in the Hausdorff topology for closed subsets of $[0, c)$ to $\sigma(M) \cap[0, c)$.

(2) Every function $f \in H^{1}(M)$ with spectral measure supported in $[0, c)$ is an effective limit of a sequence of functions $f_{i} \in H^{1}\left(M_{i}\right)$ with spectral measures supported in $[0, c)$. 
(3) For every $\lambda \in[0, c) \cap \sigma_{\text {disc }}(M)$ and every eigenfunction $f$ with eigenvalue $\lambda$ there is a sequence of eigenfunctions $f_{i}$ on $M_{i}$ with respect to eigenvalues $\lambda_{i} \in \sigma_{\mathrm{disc}}\left(M_{i}\right) \cap[0, c)$ which converge effectively to $f$.

Proof. With the assumptions in the statement of the proposition, let $R_{i} \rightarrow \infty$ be a convergence inducing sequence for our convergent sequence $\left(M_{i}, A_{i}\right)$ of manifold pairs. We choose an escaping family of sets $\Omega_{i}$ with the property that $\liminf _{i \rightarrow \infty} \mu_{1}\left(\Omega_{i}\right) \geq c>0$. Furthermore, there is a sequence $r_{i} \rightarrow \infty$ such that $\Omega_{i} \supset M-B\left(A_{i}, R_{i}-r_{i}\right)$.

For each $R>0$ the Laplacian acts on the Hilbert space $H^{1}(B(A, R))$. As $R \rightarrow \infty$ its spectrum $\sigma(B(A, R))$ converges in the Hausdorff topology for closed subsets of $[0, \infty)$ to the spectrum $\sigma(M)$ of $M$. Since there is a $\left(1+\varepsilon_{i}\right)$-bilipschitz map $F_{i}$ of $B\left(A, R_{i}\right)$ onto a neighborhood of $B\left(A_{i}, R_{i}\right)$ in $M_{i}$, this means that as $i \rightarrow \infty$ the spectrum of the Laplacian on $B\left(A_{i}, R_{i}\right)$ converges in the Hausdorff topology to the spectrum of $M$.

Let $\varepsilon>0$ and for $\delta>0$ let $\rho=\rho(\varepsilon / 2, c, \delta)$ be as in Corollary 2.4. If $i$ is sufficiently large then we have $\mu_{1}\left(\Omega_{i}\right) \geq c-\varepsilon / 2$ and $R_{i}-r_{i} \geq \rho$. By Corollary 2.4, the intersection $\sigma\left(M_{i}\right) \cap[0, c-\varepsilon]$ is contained in the $\delta$-neighborhood of $\sigma\left(B\left(A_{i}, R_{i}\right)\right)$, and $\sigma\left(B\left(A_{i}, R_{i}\right)\right) \cap[0, c-\varepsilon]$ is contained in the $\delta$-neighborhood of $\sigma\left(M_{i}\right)$. Since $\varepsilon>0$ and $\delta>0$ were arbitrary we conclude that as $i \rightarrow \infty$ (and possibly after passing to a subsequence) the spectrum of $M_{i}$ converges in the Hausdorff topology to a closed subset $B$ of $[0, \infty)$ with the property that $B \cap[0, c)=\sigma(M) \cap[0, c)$. This shows the first part of our proposition.

To show the second part, let $f$ be a function on $M$ with spectral measure supported in $[0, c-\varepsilon]$. We have to show that $f$ is an effective limit of functions on $M_{i}$ whose spectral measures converge to the spectral measure of $f$. But this follows once again from Corollary 2.4. Namely, every function $f$ on $M$ with spectral measure contained in $[0, c-\varepsilon]$ can be approximated in $H^{1}(M)$ by functions supported on $B(A, R)$ for larger and larger $R$ and with spectral measure as elements of $H^{1}(B(A, R))$ supported in $[0, c-\varepsilon / 2]$. On the other hand, for every $\kappa>0$, every function on $B(A, R)$ whose spectral measure is supported in $[0, c-\varepsilon / 2]$ admits an orthogonal decomposition into finitely many functions whose spectral measures are supported on intervals of length smaller than $\kappa$. If $\varphi \in H^{1}(B(A, R))$ is such a function and if $i>0$ is large enough that $R_{i}>R$ then we can apply Corollary 2.4 to the function $\varphi \circ F_{i}^{-1}$ on $M_{i}$ to obtain the required approximation.

We are left with showing the third part of our proposition. For this let $f$ be an eigenfunction on $M$ with eigenvalue $\lambda \in[0, c)$. Then there is a number $\delta>0$ such that the space of functions with spectral measure supported in $[\lambda-\delta, \lambda+\delta]$ is finite dimensional. Our above argument immediately implies that for sufficiently large $i$ the dimension of the space of functions on $M_{i}$ with spectral measure supported in $[\lambda-\delta / 2, \lambda+\delta / 2]$ is finite as well. This completes the proof of our proposition. 
For an integer $k \geq 1$ and a nonempty open subset $\Omega$ of a Riemannian manifold the $k$-th Rayleigh quotient $\mu_{k}(\Omega)$ of $\Omega$ is defined to be the infimum of all numbers $a>0$ with the following property. There are $k$ smooth functions $f_{1}, \ldots, f_{k}$ with compact support in $\Omega$ which are orthonormal with respect to the $L^{2}$-inner product $(f, h)_{2}=\int_{M} f h$ on $M$ and such that their gradients $\nabla f_{i}$ satisfy the inequality

$$
\mathcal{R}\left(f_{i}\right)=\int\left\|\nabla f_{i}\right\|^{2} / \int f_{i}^{2}<a .
$$

As an immediate consequence of Proposition 2.5 we obtain

Corollary 2.6. Let $\left(M_{i}, A_{i}\right)$ be a sequence of manifold pairs converging to the manifold pair $(M, A)$ and let $\Omega_{i} \subset M_{i}-A_{i}$ be a sequence of escaping sets. If $\lim \inf _{i \rightarrow \infty} \mu_{1}\left(\Omega_{i}\right) \geq \min \sigma_{\mathrm{ess}}(M)$ then $\mu_{k}\left(M_{i}\right) \rightarrow \mu_{k}(M)$ for every $k \geq 1$.

Proof. Let $v_{0} \in[0, \infty]$ be the minimum of the essential spectrum of $M$. If $v_{0}=\infty$ then our corollary is immediate from Proposition 2.5, so we may assume that $v_{0}<\infty$. Using again Proposition 2.5 it is enough to show that $\lim _{\sup _{i \rightarrow \infty}} \mu_{k}\left(M_{i}\right) \leq v_{0}$ for every fixed $k>0$. Since $v_{0}$ is contained in the essential spectrum of $M$ there is for every $k$ and every $\varepsilon>0$ an orthonormal family $f_{1}, \ldots, f_{k}$ of functions in $L^{2}(M)$ with support in a fixed compact ball $B \subset M$ and Rayleigh quotients $\mathcal{R}\left(f_{j}\right)<$ $v_{0}+\varepsilon$. For $i$ sufficiently large the set $B$ is contained in the domain of our $\left(1+\varepsilon_{i}\right)$ bilipschitz map $F_{i}$. Since $\varepsilon_{i} \rightarrow 0(i \rightarrow \infty)$ this means that for large $i$ we can find an orthonormal family $f_{1}^{i}, \ldots, f_{k}^{i}$ of functions on $M_{i}$ with $\mathcal{R}\left(f_{j}^{i}\right)<v_{0}+2 \varepsilon$. This shows that $\lim \sup _{i \rightarrow \infty} \mu_{k}\left(M_{i}\right) \leq v_{0}$.

We conclude this section with an example which illustrates how our Proposition 2.5 can be applied. We consider non-elementary torsion free Kleinian groups, i.e. finitely generated torsion free discrete subgroups of the isometry group $\operatorname{PSL}(2, \mathbb{C})$ of hyperbolic 3-space $\mathbb{H}^{3}$ which do not contain an abelian subgroup of finite index. The limit set $\Lambda$ of such a Kleinian group $\Gamma$ is the smallest closed $\Gamma$-invariant subset of the ideal boundary of $\mathbb{H}^{3}$. The closure in $\mathbb{H}^{3}$ of the convex hull of $\Lambda$ is invariant under the action of $\Gamma$ and projects to the convex core $C(M)$ of $M=\mathbb{H}^{3} / \Gamma$. A sequence $\left\{\Gamma_{i}\right\}_{i}$ of Kleinian groups converges algebraically to a Kleinian group $\Gamma$ if for almost every $i$ there is an isomorphism $\rho_{i}: \Gamma \rightarrow \Gamma_{i}$ such that $\rho_{i}(\zeta) \rightarrow \zeta$ for every fixed $\zeta \in \Gamma$. The sequence $\left\{\Gamma_{i}\right\}$ converges geometrically to $\Gamma$ if the quotient manifolds $M_{i}=\mathbb{H}^{3} / \Gamma_{i}$ converge in the pointed Lipschitz topology to $M=\mathbb{H}^{3} / \Gamma$. The sequence $\left\{\Gamma_{i}\right\}$ converges strongly to $\Gamma$ if they converge algebraically and geometrically to $\Gamma$.

A torsion free Kleinian group $\Gamma$ is called geometrically finite if the volume of the one-neighborhood of the convex core of $\mathbb{H}^{3} / \Gamma$ is finite. We then call the quotient manifold $\mathbb{H}^{3} / \Gamma$ geometrically finite as well. Generalizing earlier work of Comar and 
Taylor [CoT], Canary and Taylor show in [CT] that the bottom of the spectrum of geometrically finite hyperbolic 3-manifolds is continuous with respect to the strong topology. We state a slight extension of their main result as a corollary and show how it can be deduced from Proposition 2.5 and an observation of McMullen.

Corollary 2.7. Let $\left\{\Gamma_{i}\right\} \subset \operatorname{PSL}(2, \mathbb{C})$ be a sequence of Kleinian groups which converges strongly to a geometrically finite Kleinian group $\Gamma$. Then the intersection with $(0,1)$ of the spectrum of $\mathbb{H}^{3} / \Gamma_{i}$ converges in the Hausdorff topology to the intersection with $(0,1)$ of the spectrum of $\mathbb{H}^{3} / \Gamma$.

Proof. Let $\Gamma_{i}, \Gamma$ be as in the corollary. We write $M_{i}=\mathbb{H}^{3} / \Gamma_{i}$ and $M=\mathbb{H}^{3} / \Gamma$. Let $C\left(M_{i}\right)$ be the convex core of $M_{i}$, and for $\varepsilon>0$ let $M_{i}^{<\varepsilon}$ denote the $\varepsilon$-thin part of $M_{i}$ where the injectivity radius is less than $\varepsilon$. The truncated core is defined by $C_{\varepsilon}\left(M_{i}\right)=C\left(M_{i}\right)-M_{i}^{<\varepsilon}$. The truncated core of every geometrically finite manifold is compact.

Now if $\Gamma_{i} \rightarrow \Gamma$ strongly and if $\Gamma$ is geometrically finite then the same is true for almost all of the groups $\Gamma_{i}[\mathrm{~T}]$. Moreover, by Theorem 4.1 of [MM], for each $\varepsilon>0$ the truncated cores $C_{\varepsilon}\left(M_{i}\right)$ of the manifolds $M_{i}$ converge strongly to the truncated convex core $C_{\varepsilon}(M)$ of $M$. This means that for the $\left(1+\varepsilon_{i}\right)$-bilipschitz homeomorphisms $F_{i}: B\left(x, R_{i}\right) \subset M \rightarrow F_{i}\left(B\left(x, R_{i}\right)\right) \subset M_{i}$ as in the definition of geometric convergence and for large enough $i$ the truncated core $C_{\varepsilon}\left(M_{i}\right)$ is contained in the $\varepsilon_{i}$-neighborhood of $F_{i}\left(C_{\varepsilon}(M)\right)$.

Now it is well known (and explicitly explained in $[\mathrm{H}]$ ) that for sufficiently small $\varepsilon$ and for every $\delta>0$ there is a number $R>0$ such that for every geometrically finite manifold $N$ the smallest Raleigh quotient of $N-B\left(C_{\varepsilon}(N, R)\right)$ is not smaller than $1-\delta$. Thus we can apply Proposition 2.5 for $A=C_{\varepsilon}(M)$ and deduce that indeed the intersection of the spectrum of $M_{i}$ with $(0,1)$ (which consists of finitely many eigenvalues, compare $[\mathrm{H}]$ ) converges in the Hausdorff topology to the intersection with $(0,1)$ of the spectrum of $M$. In particular, the bottom of the spectrum of $M_{i}$ converges as $i \rightarrow \infty$ to the bottom of the spectrum of $M$.

\section{Development of cusps}

In this section let always $M$ be a closed manifold of dimension $n \geq 2$ and let $N \subset M$ be a smooth closed 2-sided hypersurface in $M$. Then there is a tubular neighborhood $U$ of $N$ which is diffeomorphic to $N \times[-1,1]$.

For $s \in[0,1]$ choose a smooth Riemannian metric $h_{s}$ on $N$ which depends smoothly on $s$ and let $\rho:(0,1] \times[-1,1] \cup\{0\} \times([-1,0) \cup(0,1]) \rightarrow(0, \infty)$ be a smooth function. Using the metrics $\rho(s, t) h_{s}$ on $N$ we define for each $s>0$ a smooth metric $g_{s}$ on $N \times[-1,1]$ by $g_{s}=\frac{1}{t^{2}+s^{2}} d t^{2}+\rho(s, t) h_{s}$. As $s \searrow 0$ these 
metrics converge uniformly on compact subsets of $N \times([-1,0) \cup(0,1])$ to a complete metric $g_{0}$. We assume that the metrics $g_{s}$ can be extended to smooth Riemannian metrics on $M-U$ which depend smoothly on $s \in[0,1]$. We denote these metrics again by $g_{s}$, and we write $M_{s}$ for the manifold $M$ with the metric $g_{s}$ (for $s=0$ we replace $M$ by $M-N$ ). We allow $M-N$ to be disconnected.

Lemma 3.1. The manifold pairs $\left(M_{s}, M_{s}-U\right)$ converge as $s \rightarrow 0$ to the manifold $\operatorname{pair}\left(M_{0}, M_{0}-U\right)$.

Proof. By construction, the distance in $M_{0}$ between the subsets $M_{0}-U$ and $N \times$ $([-\delta, 0) \cup(0, \delta])$ goes to infinity as $\delta \searrow 0$. Since by our hypothesis the metrics $\rho(s, t) h_{s}$ on $N$ converge as $s \searrow 0$ locally uniformly in $t \in[-1,0) \cup(0,1]$ to the metrics $\rho(0, t) h_{0}$ our lemma follows.

Example 3.2. Let $M$ be a smooth connected noncompact orientable $n$-dimensional hyperbolic manifold of finite volume. Then $M$ has a finite number $k \geq 1$ of standard cusps. These cusps are given by a two-sided closed embedded hypersurface $N \subset M$ which consists of $k$ connected components and divides $M$ into a manifold $\bar{M}$ and the cusps $E_{1}, \ldots, E_{k}$. The metric $h$ on $N$ induced from the hyperbolic metric is flat and therefore $N$ is a finite quotient of a collection of $k$ tori of dimension $n-1$. The union $\cup_{i=1}^{k} E_{i}$ of our ends $E_{1}, \ldots, E_{k}$ is diffeomorphic to $N \times[0, \infty)$ and carries the warped product metric $d t^{2}+e^{-2 t} h$.

Choose a fixed smooth convex function $\alpha: \mathbb{R} \rightarrow(0, \infty)$ with the property that $\alpha(t)=e^{-t}$ for $t \leq 0, \alpha(t)=e^{-1}$ for large $t$ and such that $\alpha^{\prime} \geq-\alpha$ and $\alpha^{\prime \prime} \leq \alpha$. For each fixed $s \in(0,1]$ define a new metric $g_{s}$ on $N \times[0, \infty)$ by $g_{s}=d t^{2}+s^{2} \alpha(t+$ $\log s)^{2} h$. Then the metric $g_{s}$ coincides with the hyperbolic metric on $N \times[0,-\log s]$ and extends to a complete smooth metric on all of $M$ which coincides with the hyperbolic metric on $\bar{M}$. We denote this metric again by $g_{s}$. The sectional curvature of $g_{s}$ is contained in $[-1,0]$. There is a number $\tau_{0}>0$ not depending on $s$ such that the restriction of $g_{s}$ to $N \times\left[-\log s+\tau_{0} / 2, \infty\right)$ is the flat product metric $e^{-1} s^{2} h \times[0, \infty)$.

Write $E_{s}=N \times\left(-\log s+\tau_{0}, \infty\right)$. We can glue two copies of $M-E_{s}$ along the boundary with the natural isometry between the two boundary manifolds $N \times$ $\left\{-\log s+\tau_{0}\right\}$ to obtain a compact connected Riemannian manifold $M_{s}$. This manifold contains two isometric copies of $\bar{M}$ and a totally geodesic embedded flat hypersurface which corresponds to the boundary components of the ends $E_{s}$. If we denote by $A_{s}$ the union of our two copies of $\bar{M}$ in $M_{s}$ then as $s \searrow 0$ the manifold pairs $\left(M_{S}, A_{s}\right)$ converge in the Lipschitz topology to the disconnected hyperbolic manifold pair $\left(M_{0}, A_{0}\right)$ which consists of two copies of the pair $(M, \bar{M})$. We call such a converging sequence of manifolds a standard cusp convergence. With respect to a suitable parametrization of the cylinders $M_{s}-A_{s}$ in $M_{s}$ our family of metrics can be represented as a 1parameter family of warped product metrics of the above form. 
Recall that the bottom of the spectrum of a standard hyperbolic cusp of dimension $n$ equals $(n-1)^{2} / 4$. From Proposition 2.5 we therefore obtain that as $s \searrow 0$ the intersection with $\left(0,(n-1)^{2} / 4\right)$ of the spectrum of $M_{s}$ converges to the intersection with $\left(0,(n-1)^{2} / 4\right)$ of the spectrum of $M_{0}$. Since $M_{0}$ is disconnected and each of its two components is of finite volume, the eigenvalue 0 of $M_{0}$ has multiplicity 2 and therefore the second eigenvalue of the manifolds $M_{s}$ converges as $s \searrow 0$ to 0 .

Assume from now on that the second eigenvalue of the metric $\rho(s, t) h_{s}$ on $N$ goes to $\infty$ as $(s, t) \rightarrow(0,0)$. Since the metrics $h_{s}$ are defined for every $s \in[0,1]$ this is equivalent to requiring that our function $\rho$ extends continuously to 0 at $(0,0)$. Notice that the volume of $M_{0}$ may be infinite.

Let $v_{s}(t)$ be the volume element of the metric $\rho(s, t) h_{s}$ on $N$. For $s \in[0,1]$ let $W_{s} \subset H^{1}\left(M_{S}\right)$ be the closure in $H^{1}\left(M_{S}\right)$ of the space of smooth functions $f$ on $M_{S}$ which satisfy $\int_{N \times\{t\}} f d v_{s}(t)=0$ for all $t \in[-1 / 2,1 / 2]$. Denote by $\mu_{s}$ the volume element of the metric $g_{s}$ on $M$. In the sequel we write $\int\|\nabla f\|^{2} d \mu_{s}$ to denote the integral of the square norm of the differential of $f$ with respect to the metric $g_{s}$.

Lemma 3.3. For every $\varepsilon>0, c>0$ there exists a number $\delta=\delta(\varepsilon, c)>0$ with the following property. Let $s \leq \delta$ and let $f \in W_{s}$ be a function with

$$
\int_{N \times[-1 / 2,1 / 2]}\|\nabla f\|^{2} d \mu_{s}<c \int f^{2} d \mu_{s}
$$

Then we have

$$
\int_{N \times[-\delta, \delta]} f^{2} d \mu_{s}<\varepsilon\|f\|^{2} .
$$

In particular, the Hilbert space $W_{s} \subset H^{1}\left(M_{s}\right)$ is compactly embedded in $L^{2}\left(M_{S}\right)$.

Proof. Let $\mu_{2}(s, t)$ be the second Rayleigh quotient of the metric $\rho(s, t) h_{s}$ on $N$. By our assumption we have $\mu_{2}(s, t) \rightarrow \infty$ as $(s, t) \rightarrow(0,0)$ and therefore for every $k>0$ there is a number $\tau=\tau(k) \in(0,1 / 2)$ such that $\mu_{2}(s, t)>k$ for all $s<\tau$, all $t$ with $|t|<\tau$.

Now if $f \in W_{s}$ then for every $t \in[-1 / 2,1 / 2]$ the restriction of $f$ to $N \times\{t\}$ is orthogonal to the constant functions. Moreover the measure $\mu_{s}$ can be represented in the form $d v_{s}(t) \times \alpha(s, t) d t$ for a smooth function $\alpha \geq 1$. Consequently for $s<\tau$ we have

$$
\begin{aligned}
\int_{N \times[-1 / 2,1 / 2]}\|\nabla f\|^{2} d \mu_{s} & \geq \int_{-1 / 2}^{1 / 2}\left(\int_{N \times\{t\}} \mu_{2}(s, t) f^{2} d v_{s}(t)\right) d t \\
& \geq k \int_{N \times[-\tau, \tau]} f^{2} d \mu_{s} .
\end{aligned}
$$


If $\int_{N \times[-1 / 2,1 / 2]}\|\nabla f\|^{2} d \mu_{s}<c \int f^{2} d \mu_{s}$ for some $c>0$ then we deduce from this that $\int_{N \times[-\tau, \tau]} f^{2} d \mu_{s}<\frac{c}{k} \int f^{2} d \mu_{s}$ which shows the first part of our lemma. Compactness of the embedding $W_{S} \subset H^{1}\left(M_{S}\right) \rightarrow L^{2}\left(M_{S}\right)$ then follows from standard compactness results.

The following proposition generalizes an earlier result of Judge $[\mathrm{J}]$ and shows our Theorem B. Its proof uses the ideas of Judge [J], with our simple Lemma 3.3 as the main new ingredient. We include the (rather short) proof for the sake of completeness. In contrast to Section 2 we now mean by an eigenfunction a solution of an equation $\Delta-\lambda=0$ for some $\lambda \in \mathbb{R}$ which is not required to be square integrable.

Proposition 3.4. Assume that $\rho(s, t) \searrow 0$ as $(s, t) \rightarrow 0$. Let $c>0$ and let $\left\{s_{i}\right\}_{i} \subset$ $(0,1]$ be a sequence converging to 0 . Let $f_{i}$ be an eigenfunction on $M_{s_{i}}$ with respect to an eigenvalue $\lambda_{i} \leq c$. Then up to passing to a subsequence and renormalization, the functions $f_{i}$ converge locally uniformly on $M-N$ to a nontrivial eigenfunction $f$ on $M_{0}$ with respect to the eigenvalue $\lambda_{0}=\lim _{i \rightarrow \infty} \lambda_{i}$.

Proof. Define a linear projection $P_{s}: L^{2}\left(U \subset M_{s}\right) \rightarrow L^{2}\left(U \subset M_{s}\right)$ by

$$
P_{s} f(x, t)=\int_{N \times\{t\}} f d v_{s}(t) .
$$

In other words, $P_{s} f$ is the function which is obtained by integration of $f$ along the manifolds $M \times\{t\}$ with respect to the volume form of the metric $\rho(s, t) h_{s}$.

For $i \geq 0$ let $f_{i}$ be an eigenfunction on $M_{s_{i}}$ with respect to the eigenvalue $\lambda_{i}$. We assume that these eigenvalues are bounded from above by some $c>0$. Let $\delta=\delta(1 / 2,2 c)$ be as in Lemma 3.3. Using an idea of Judge [J] we define

$$
\tilde{f}_{i}(x, t)= \begin{cases}f_{i}(x, t), & \text { if }|t| \geq \delta \\ \left(f_{i}-P_{s_{i}} f_{i}\right)(x, t), & \text { otherwise }\end{cases}
$$

To simplify our notation we assume that the functions $\tilde{f}_{i}$ are normalized; this only depends on the normalization of $f_{i}$.

Let $\alpha:(-1,1) \rightarrow[0,1]$ be a smooth function supported in $[-3 / 4,3 / 4]$ with $\alpha(t)=1$ for $t \in[-5 / 8,5 / 8]$ and define $u_{i}(x, t)=f_{i}(x, t)-\alpha(t) P_{s_{i}} f_{i}(x, t)$. By our normalization assumption the $L^{2}$-norm of the function $u_{i}$ is not bigger than 1 , moreover $u_{i}$ is contained in $W_{s_{i}}$.

We claim that the $L^{2}$-norm of the gradient of $u_{i}$ is bounded independent of $i$. To see this recall that our metrics $g_{s}$ are warped product metrics on $N \times[-1,1]$ and therefore for each fixed $s \in(0,1], t \in(-1,1)$ and every smooth function $\varphi$ on $M_{s}$ we have $\int_{N \times\{t\}} g_{s}\left(\nabla\left(\varphi-P_{s} \varphi\right), \nabla\left(P_{s} \varphi\right)\right) d v_{s}(t)=0$. Namely, since our metric is a warped product the normalized volume forms of the metrics $\rho(s, t) h_{s}$ on $N$ are 
independent of $t \in(-1,1)$. This implies that the gradient $\nabla\left(P_{S} \varphi\right)$ of $P_{S} \varphi$ is of the form $\nabla\left(P_{s} \varphi\right)(x, t)=\zeta(t) \frac{\partial}{\partial t}$ where $\zeta$ only depends on $t$ and $\nabla\left(\varphi-P_{s} \varphi\right)=X+\chi \frac{\partial}{\partial t}$ where $\int_{N \times\{t\}} \chi d v_{s}(t)=0$ and $X$ is tangent to the first factor foliation of $N \times[-1,1]$.

Let $\beta:(-1,1) \rightarrow[0,1]$ be a smooth function with compact support which equals 1 on $[-3 / 4,3 / 4]$. Define $v_{i}(x, t)=\beta(t)\left(f_{i}-P_{s_{i}} f_{i}\right)(x, t)$; then $\left\|v_{i}\right\|^{2} \leq 1$. Since $f_{i}$ is an eigenfunction with respect to the eigenvalue $\lambda_{i}$, by the definition of $v_{i}$ and the above we have

$$
\begin{aligned}
\lambda_{i} & \geq \lambda_{i} \int v_{i} u_{i} d \mu_{s_{i}}=\lambda_{i} \int v_{i} f_{i} d \mu_{s_{i}}=\int g_{s_{i}}\left(\nabla v_{i}, \nabla f_{i}\right) d \mu_{s_{i}} \\
& \geq \int_{N \times[-3 / 4,3 / 4]}\left\|\nabla\left(f_{i}-P_{s_{i}} f_{i}\right)\right\|^{2} d \mu_{s_{i}} \geq \int_{N \times[-5 / 8,5 / 8]}\left\|\nabla u_{i}\right\|^{2} d \mu_{s_{i}} .
\end{aligned}
$$

Notice that this estimate relies in a crucial way on the fact that the normalized volume element of $\rho(s, t) h_{s}$ is independent of $t$.

On the other hand, let $\tilde{\beta}:[-1,1] \rightarrow[0,1]$ be a smooth function supported in $[-1,1 / 2] \cup[1 / 2,1]$ which is constant 1 on $[-1,-5 / 8] \cup[5 / 8,1]$. Write $\tilde{v}(x, t)=$ $\tilde{\beta}(t) f_{i}(x, t)$. As before we deduce that

$$
\lambda_{i} \geq \lambda_{i} \int \tilde{v}_{i} f_{i} d \mu_{s_{i}} \geq \int_{M-N \times[-5 / 8,5 / 8]}\left\|\nabla f_{i}\right\|^{2} d \mu_{s_{i}} .
$$

Now for $5 / 8 \leq|t| \leq 1$ we have

$$
\nabla u_{i}(x, t)=\nabla f_{i}(x, t)-\alpha^{\prime}(t) P_{s_{i}} f_{i} \frac{\partial}{\partial t}-\alpha(t) \nabla\left(P_{s_{i}} f_{i}\right)
$$

and therefore there is a constant $a>0$ not depending on $i$ such that

$$
\begin{aligned}
& \int_{M-N \times[-5 / 8,5 / 8]}\left\|\nabla u_{i}\right\|^{2} d \mu_{s_{i}} \\
& \quad \leq a \int_{M-N \times[-5 / 8,5 / 8]}\left\|\nabla f_{i}\right\|^{2} d \mu_{s_{i}}+a \int_{N \times[-1,-5 / 8] \cup[5 / 8,1]} f_{i}^{2} d \mu_{s_{i}} .
\end{aligned}
$$

From this and inequality (1) above we conclude that the $L^{2}$-norm of the gradient of $u_{i}$ is bounded independent of $i$.

We claim that after passing to a subsequence the functions $u_{i}$ converge in the space of locally square integrable functions on $M_{0}$ to a function $u_{0}$ with $\left\|u_{0}\right\|^{2}=$ $\lim _{i \rightarrow \infty}\left\|u_{i}\right\|^{2} \leq 1$. This is obvious if the $L^{2}$-norms of the functions $u_{i}$ converge to 0 as $i \rightarrow \infty$, so assume that there is some $c>0$ such that $\left\|u_{i}\right\|^{2} \geq c$ for all $i$. Since the $L^{2}$-norm of the gradient of $u_{i}$ is bounded independent of $i$, the Raleigh quotients of $u_{i}$ are bounded independent of $i$. Lemma 3.3 then shows that after passing to a subsequence we may assume that the functions $u_{i}$ converge locally in $L^{2}\left(M_{0}\right)$ to a function $u_{0}$ with $\left\|u_{0}\right\|^{2}=\lim _{i \rightarrow \infty}\left\|u_{i}\right\|^{2}$. 
Next we observe that after passing to a another subsequence we may assume that the restrictions to $N \times([-1,-\delta] \cup[\delta, 1])$ of the functions $\tilde{f}_{i}-u_{i}$ converge in $L^{2}\left(M_{0}\right)$ to a function $\chi$. Again this is obvious if the $L^{2}$-norm of $\tilde{f}_{i}-u_{i}$ tends to 0 with $i$. Otherwise observe that the function $\tilde{f}_{i}-u_{i}$ can be viewed as a function on $[-1,-\delta] \cup[\delta, 1]$. Its $L^{2}$-norm with respect to a measure which is uniformly equivalent to the standard Lebesgue measure is at most 1 . Our above consideration implies that the $L^{2}$-norms of the derivatives of $\tilde{f}_{i}-u_{i}$ are bounded independent of $i$. Thus we obtain convergence from compactness of the embedding $H^{1}(I) \rightarrow L^{2}(I)$ for a compact interval $I \subset \mathbb{R}$. In particular, the functions $\tilde{f}_{i}$ converge in $L^{2}\left(M_{0}\right)$ to the function $u_{0}+\chi$.

Consider again inequality (1) above. By Lemma 3.3 and our choice of $\delta$ we either have $\left\|u_{i}\right\|^{2}<1 / 2$ or $\int_{N \times[-\delta, \delta]} u_{i}^{2} d \mu_{s_{i}}<\int u_{i}^{2} d \mu_{s_{i}} / 2$ for all sufficiently large $i$. In both cases we conclude that $\int_{N \times[-\delta, \delta]} u_{i}^{2} d \mu_{s_{i}} \leq 1 / 2$ for large $i$. Thus our function $u_{0}$ necessarily satisfies $\int_{N \times[-\delta, 0) \cup(0, \delta]} u_{0}^{2} d \mu_{0} \leq 1 / 2$. Since the function $\chi$ is supported in $M-N \times[-\delta, \delta]$ and $\left\|\chi+u_{0}\right\|^{2}=1$ we conclude that after passing to a subsequence the restrictions to $M-N \times[-\delta, \delta]$ of the functions $f_{i}$ converge in $L^{2}\left(M_{0}\right)$ to a function $f_{0}$ with $\left\|f_{0}\right\|^{2} \in[1 / 2,1]$.

The function $f_{i}$ is a solution of an elliptic equation with smooth coefficients. With respect to the reference metric $g_{0}$ on $M-N \times(-\delta / 2, \delta / 2)$ the $C^{2}$-norms of these coefficients are uniformly bounded. Since the $L^{2}$-norms of the restrictions to $M_{s_{i}}-N \times[-\delta, \delta]$ of the functions $f_{i}$ are uniformly bounded as well, standard elliptic theory implies that for every $\varepsilon>0$ there is a constant $c(\varepsilon)>0$ which bounds the $C^{2}$-norm of the restriction of $f_{i}$ to $M-N \times[-\delta-\varepsilon, \delta+\varepsilon]$. Thus after passing to a subsequence the functions $f_{i}$ converge locally uniformly on $M-N \times[-\delta, \delta]$ to $f_{0}$. This implies that for $\lambda_{0}=\lim _{i \rightarrow \infty} \lambda_{i}$ the function $f_{0}$ is a nontrivial solution of the differential equation $\Delta_{0}-\lambda_{0}=0$.

Our above argument also shows that the function $f_{0}$ is the restriction to $M-N \times$ $[-\delta, \delta]$ of an eigenfunction on $M_{0}$ which is a locally uniform limit of a subsequence of our functions $f_{i}$. Namely, for $k>-\log \delta+\log 2$ define

$$
\tilde{f}_{i, k}(x, t)= \begin{cases}f_{i}(x, t), & \text { if }|t| \geq 2^{-k}, \\ \left(f_{i}-P_{s_{i}} f_{i}\right)(x, t) & \text { otherwise }\end{cases}
$$

and write $a_{i, k}=1 /\left\|\tilde{f}_{i, k}\right\|$. For each fixed $i$ the sequence $\left\{a_{i, k}\right\}_{k}$ is monotonously decreasing. As before we conclude that after passing to a subsequence the restrictions of $a_{i, k} f_{i, k}$ to $M-N \times\left[2^{-k}, 2^{-k}\right]$ converge locally uniformly to a solution $\bar{f}_{0, k} \not \equiv 0$ of the equation $\Delta_{0}-\lambda_{0}=0$. Its restriction to $M-N \times\left[-2^{-k}, 2^{-k}\right]$ necessarily coincides with a nonnegative multiple of $\bar{f}_{0, k-1}$. Since no nontrivial solution of the equation $\Delta_{0}-\lambda_{0}=0$ can vanish on a nontrivial open set the restriction of our function to $M-N \times\left[-2^{-k}, 2^{-k}\right]$ is in fact a positive multiple of $\bar{f}_{0, k-1}$. With a standard diagonal sequence argument we conclude from this that after passing to a 
subsequence our eigenfunctions $f_{i}$ converge locally uniformly to an eigenfunction $f_{0}$ on $M_{0}$.

The following example shows that the limit function obtained in Proposition 3.4 is in general not square integrable, even if the curvature of all our manifolds as well as their volumes are uniformly bounded.

Example 3.5. Consider a closed hyperbolic surface $S$ of genus 2 which consists of two bordered tori $T_{1}, T_{2}$ glued at the boundary. Choose a simple closed geodesic $\gamma$ on $T_{1}$ which cuts $T_{1}$ into a pair of pants. We denote by $g_{s}$ the hyperbolic metric on $S$ which we obtain by leaving the torus $T_{2}$ and the twist parameters for the glueings fixed and replacing the torus $T_{1}$ by a torus for which the length of the geodesic $\gamma$ equals $s$. For a fixed point $q \in T_{2}$ the pointed surfaces $\left(\left(S, g_{s}\right), q\right)$ degenerate as $s \searrow 0$ to a twice punctured hyperbolic torus $\left(S_{0}, g_{0}\right)$ with two finite volume cusps. The essential spectrum of $S_{0}$ is bounded from below by $1 / 4$ and the second Rayleigh quotient $\mu_{2}\left(S_{0}\right)$ of $S_{0}$ is positive. The metrics $g_{s}$ are warped product metrics in a tubular neighborhood of $\gamma$.

Choose a number $k>0$ such that there is a smooth nontrivial compactly supported function $f$ on the interval $(0, k)$ satisfying $\int_{0}^{k} f=0$ and $\int_{0}^{k}\left(f^{\prime}\right)^{2}<\mu_{2}\left(S_{0}\right) \int_{0}^{k} f^{2} / 2$. For $a>0$ and $\tau \in[0, k]$ denote by $C_{a, \tau}$ the cylinder $S^{1} \times[0, \tau]$ with the metric $a^{2} d s^{2}+d t^{2}$ (where $d s^{2}$ is the length element of total length 1 on $S^{1}$ ). For every $a>0$ the function $f$ can be viewed as a function on the cylinder $C_{a, k}$ which only depends on the second coordinate. We have $\int_{C_{a, k}} f=0$ and $\int_{C_{a, k}}\|\nabla f\|^{2}<\mu_{2}\left(S_{0}\right) \int_{C_{a, k}} f^{2} / 2$ for all $a>0$.

For $s \in(0,1]$ and $\tau \in[0, k]$ we replace the metric $g_{s}$ near $\gamma$ by a metric $\tilde{g}_{s, \tau}$ which is obtained from $g_{s}$ by cutting $S$ open along $\gamma$ and inserting the cylinder $C_{s, \tau}$. We slightly modify the resulting metric near the boundary of $C_{s, \tau}$ in such a way that we obtain a smooth metric $\tilde{g}_{s, \tau}$ depending smoothly on $s, \tau$ and such that $\tilde{g}_{s, 0}=g_{s}$. We may assume that there is an tubular neighborhood $Z \sim S^{1} \times[-1,1]$ about $\gamma$ in $S$ such that the restrictions of the metrics $\tilde{g}_{s, \tau}$ to $Z$ are warped product metrics. The metrics can be constructed in such a way that their curvature is contained in $[-1,0]$ and that their volumes are uniformly bounded.

For fixed $s>0$, the second Rayleigh quotient of $\tilde{g}_{s, \tau}$ depends continuously on $\tau \in[0, k]$. For $\tau=k$ this Rayleigh quotient is not bigger than $\mu_{2}\left(S_{0}\right) / 2$. Moreover, if $s$ is sufficiently small then the second Rayleigh quotient of $\tilde{g}_{s, 0}$ equals at least $3 \mu_{2}\left(S_{0}\right) / 4$ [CC2]. Thus there is some $\tau(s) \in[0, k]$ such that this Rayleigh quotient equals exactly $\mu_{2}\left(S_{0}\right) / 2$. We may assume that $\tau(s)$ depends continuously on $s$. Define $h_{s}=\tilde{g}_{s, \tau(s)}$. Then there is an eigenfunction $\varphi_{s}$ on $\left(S, h_{s}\right)$ with respect to the eigenvalue $\mu_{2}\left(S_{0}\right) / 2$. Moreover the metrics $h_{s}$ satisfy the assumptions in Proposition 3.4.

By Proposition 3.4, after renormalization and passing to a subsequence we may 
assume that the eigenfunctions $\varphi_{s}$ converge uniformly on compact sets to an eigenfunction $\varphi$ on $S_{0}$ with respect to the eigenvalue $\mu_{2}\left(S_{0}\right) / 2$. But then $\varphi$ can not be square integrable.

Remark. The considerations in Example 3.5 can also be used to construct for every noncompact hyperbolic surface $S$ of finite volume and every $\lambda \in\left(0, \mu_{2}(S)\right)$ an eigenfunction $\varphi$ on $S$ with respect to the eigenvalue $\lambda$. This function $\varphi$ is not square integrable.

\section{Manifolds with controlled spectral properties}

In this section we apply the results from Section 2 to construct complete Riemannian manifolds of an arbitrary dimension $n \geq 2$, with curvature in the interval $[-1,0]$, infinite volume, nonempty essential spectrum, infinitely many eigenvalues below the essential spectrum and with arbitrarily large multiplicities of an arbitrary finite number of eigenvalues. In the case $n=2$ we can choose our manifolds to be of constant curvature -1 .

Our manifolds will be constructed from building blocks which consist of complete manifolds of curvature contained in $[-1,0]$ with a fixed even number $2 k \geq 2$ of standard constant curvature cusps as in Example 3.2. We describe these building blocks in the next lemma which is a modified version of Example 4.1 of [BCD].

Lemma 4.1. For every $n \geq 2, k \geq 1$ there is a complete $n$-dimensional Riemannian manifold $X$ of infinite volume with the following properties.

(1) The curvature of $X$ is contained in $[-1,0]$.

(2) $X$ has $2 k$ standard cusps of curvature -1 which are mutually isometric.

(3) The essential spectrum $\sigma_{\mathrm{ess}}(X)$ of $M$ is not empty, and there are infinitely many different eigenvalues below $\sigma_{\mathrm{ess}}(X)$

Proof. Let $\Gamma \subset \mathrm{SO}(n, 1)$ be a non-uniform lattice. Then $V_{0}=\mathbb{H}^{n} / \Gamma$ is a hyperbolic manifold of finite volume with at least one end $C$. This end is a standard cusp.

The group $\Gamma$ is residually finite and therefore there is a finite covering $V_{1}$ of $V_{0}$ such that the cusp $C$ has at least $2 k$ preimages in $V_{1}$. We choose $2 k$ of these preimages and label them by $C_{1}, \ldots, C_{2 k}$. If $C_{1}, \ldots, C_{2 k}$ are the only cusps of $V_{1}$ then we define $N_{0}=V_{1}$.

Otherwise, i.e. if $V_{1}$ has additional cusps, then we modify the metric on each of these additional cusps as in Example 3.2. These cusps then become flat cylinders which we cut along a totally geodesic hypersurface. We obtain a manifold $V_{1}^{\prime}$ with $2 k$ cusps and a finite number of totally geodesic boundary components. Choose a second copy $V_{1}^{\prime \prime}$ of $V_{1}^{\prime}$ and glue $V_{1}^{\prime \prime}$ to $V_{1}^{\prime}$ along corresponding boundary components. The 
resulting manifold $V_{2}$ is connected, its curvature is contained in the interval $[-1,0]$ and it has precisely $4 k$ mutually isometric ends which are the cusps $C_{1}, \ldots, C_{2 k}$ of $V_{1}$ and the corresponding cusps $C_{1}^{\prime \prime}, \ldots, C_{2 k}^{\prime \prime}$ of $V_{1}^{\prime \prime}$. Since the cusps $C_{i}^{\prime \prime}$ are mutually isometric we can replace them as before by isometric cylindrical ends which we cut and glue in pairs to $k$ compact handles. We obtain a manifold $N_{0}$ with precisely $2 k$ ends. It carries a complete Riemannian metric of finite volume with curvature in the interval $[-1,0]$ in such a way that each of its ends is isometric to a fixed standard cusp of constant curvature -1 .

Let $F_{k}$ be the free group with $k$ generators $\gamma_{1}, \ldots, \gamma_{k}$. We label each of the $2 k$ ends of $N_{0}$ by one of elements $\gamma_{1}, \ldots, \gamma_{k}, \gamma_{1}^{-1}, \ldots, \gamma_{k}^{-1}$ of $F_{k}$. For $s>0$ replace the standard cusps of $N_{0}$ by a compact end with boundary equipped with the metric $g_{s}$ from Example 3.2. The resulting manifold $N_{s}$ has $2 k$ totally geodesic boundary components which we label as before by the generators of $F_{k}$. Choose one copy of $N_{s}$ for every element of $F_{k}$ and label it by this group element. Glue the boundary component with label $\gamma_{i}$ of the copy of $N_{s}$ with label $\psi \in F_{k}$ to the boundary component with label $\gamma_{i}^{-1}$ of the copy of $N_{s}$ with label $\gamma_{i} \psi$ with the obvious isometry. We obtain a smooth manifold $M$ with a complete Riemannian metric $g_{s}$ of curvature contained in $[-1,0]$ and which depends smoothly on $s \in(0,1]$. The free group $F_{k}$ acts freely and properly discontinuously on $M$ by right translations on the labels of our basic building blocks. The metrics $g_{s}$ are invariant under this action. The quotient $\left(M, g_{s}\right) / F_{k}$ is compact and can be obtained from $N_{s}$ by glueing pairwise the boundary components.

Since $\left(M, g_{s}\right)$ admits a free and properly discontinuous isometric action of $F_{k}$ with a compact quotient, the discrete spectrum of $\left(M, g_{s}\right)$ vanishes and its essential spectrum is bounded away from 0 (see the discussion in Example 4.1 of [BCD]). The bottom $v_{s}$ of this essential spectrum depends continuously on $s$ and goes to 0 as $s \rightarrow 0$.

Following [BCD] we fix a number $\tau>0$ such that $\nu_{\tau}<(n-1)^{2} / 4$ and a sequence $\tau_{i} \subset(0, \tau)$ such that $\tau_{i}<\tau_{j}$ and $\nu_{\tau_{i}}<\nu_{\tau_{j}}$ for $i<j$. We use this sequence to construct inductively our building block.

There is a natural word norm on the group $F_{k}$ defined by our choice of generators. For $m \geq 1$ we denote by $B(m)$ the connected submanifold of $M$ which consists of precisely those copies of our manifold $N_{s}$ which are labeled by elements of $F_{k}$ of word norm at most $m$. Then $B(m)$ is a smooth submanifold of $M$ with boundary. Each of its boundary components is totally geodesic with respect to $g_{s}$. The set $B(0)$ is just the copy of $N_{s}$ which corresponds to the unit element in $F_{k}$.

In our first step we determine a number $m_{1}>0$ such that there is a function $\psi_{1}$ on $\left(M, g_{\tau_{1}}\right)$ which is supported in $B\left(m_{1}-1\right)-B(1)$ and with Rayleigh quotient $\mathcal{R}\left(\psi_{1}\right)<v_{\tau_{2}}$. Modify the metric of $B\left(m_{1}\right)$ near the boundary so that the new metric coincides with $g_{\tau_{1}}$ on $B\left(m_{1}-1\right)$ and with $g_{\tau_{2}}$ near the boundary. Glue the resulting manifold along its boundary to $\left(M, g_{\tau_{2}}\right)-B\left(m_{1}\right)$. We obtain a new manifold $\tilde{M}_{1}$. 
Since the essential spectrum of any Riemannian manifold does not change under a compactly supported change of the metric, the bottom of the essential spectrum of $\tilde{M}_{1}$ equals $v_{\tau_{2}}$. But the Rayleigh quotient of the function $\psi_{1}$ on $B\left(m_{1}-1\right) \subset \tilde{M}_{1}$ is smaller than $v_{\tau_{2}}$ and hence $\tilde{M}_{1}$ has an eigenvalue below its essential spectrum.

We can now iterate this construction. In our $i$-th step we begin with a metric $\tilde{g}_{i}$ on $M$ which coincides with the metric $g_{\tau_{i}}$ on $M-B\left(m_{i}\right)$ for some $m_{i}>0$ and such that there are $i$ functions on $\left(M, \tilde{g}_{i}\right)$ with pairwise disjoint support contained in $B\left(m_{i}-1\right)-B(1)$ and with Rayleigh quotients smaller than $v_{\tau_{i}}$. There are at least $i$ distinct eigenvalues below the essential spectrum. Choose a function $\psi_{i+1}$ supported on $B\left(m_{i+1}-1\right)-B\left(m_{i}\right)$ for some $m_{i+1}>m_{i}$ with Rayleigh quotient smaller than $v_{\tau_{i+1}}$. As before we change the metric outside $B\left(m_{i+1}\right)$ to $g_{\tau_{i+1}}$ to obtain a new metric $\tilde{g}_{i+1}$ which admits at least $i+1$ distinct eigenvalues below the essential spectrum.

We can repeat this construction infinitely often to obtain a complete manifold $X_{0}$ with infinitely many eigenvalues below the essential spectrum. The lower bound $v_{0}$ of the essential spectrum of $X_{0}$ is strictly smaller than $(n-1)^{2} / 4$.

Remove $B(0)$ from $X_{0}$ and replace it by a manifold with $2 k$ standard cusps. We claim that the complete Riemannian manifold $X$ which we obtain in this way has the properties stated in our lemma. To see this recall that the bottom of the essential spectrum of a standard hyperbolic cusp equals $(n-1)^{2} / 4>v_{0}$. Since the essential spectrum of a complete Riemannian manifold equals the essential spectrum of its ends, the bottom of the essential spectrum is $\nu_{0}$. The functions on $X_{0}$ which we constructed above are supported outside $B(1)$ and hence can be viewed as functions on $X$. This implies that there are infinitely many distinct eigenvalues below the bottom of the essential spectrum on $X$.

Using pairs of pants as in [BCD] it is clear that for $n=2$ we can choose our manifold to have constant curvature -1 .

Consider now an arbitrary finite group $\Gamma$. We call $\Gamma$ admissible if $\Gamma$ can be generated by elements of order at least 3. A set of generators $\gamma_{1}, \ldots, \gamma_{2 k}$ of $\Gamma$ is called admissible if it consists of elements of order at least 3, contains with each element also its inverse and is minimal with this property.

Let $G$ be the Cayley graph for $\Gamma$ with respect to our generators. Then $G$ is a finite connected graph whose vertices correspond to the elements of $\Gamma$. By our choice of generators the graph $G$ is simple (i.e. no multiple edges and no loops) and $2 k$-regular [dH]. Two vertices $a, b \in \Gamma$ of $G$ are connected by an edge if and only if there is some $i$ such that $b=\gamma_{i} a$. Right multiplication in $\Gamma$ induces an action of $\Gamma$ as a group of automorphisms of $G$ which is transitive on the vertices.

Assume that $\gamma_{2 i}=\gamma_{2 i-1}^{-1}$ for $1 \leq i \leq k$. Let $X$ be a manifold as in Lemma 4.1 with $2 k$ standard cusps. We label each of these cusps by one of our generators $\gamma_{i}$ of $\Gamma$. For a $k$-tuple $a=\left(s_{1}, \ldots, s_{k}\right) \in[0,1]^{k}$ we construct a complete Riemannian manifold $M(a)$ as follows: Choose $|\Gamma|$ copies of $X$ and label each of these copies 
with a different element of $\Gamma$. For $1 \leq i \leq k$ replace the standard cusps of $X$ which are labeled by $\gamma_{2 i-1}, \gamma_{2 i}$ by a compact end with boundary equipped with the metric $g_{s_{i}}$. The boundary components of the resulting manifold correspond to our generators $\gamma_{1}, \ldots, \gamma_{2 k}$. Glue the boundary component $\gamma_{i}$ of the copy of $X$ with label $\psi \in \Gamma$ to the boundary component $\gamma_{i}^{-1}$ of the copy of $X$ with label $\gamma_{i} \psi$ by the obvious isometry as before. We obtain a complete Riemannian manifold $M\left(s_{1}, \ldots, s_{k}\right)$ which consists of $|\Gamma|$ copies of $X$ glued at their boundaries. It contains a distinguished collection of totally geodesic embedded hypersurfaces, and its curvature is contained in $[-1,0]$. The essential spectrum of $M\left(s_{1}, \ldots, s_{k}\right)$ is bounded away from 0 and there are infinitely many eigenvalues below the essential spectrum. The manifold $M(0)$ consists of $|\Gamma|$ copies of $X$. We call the manifold $M\left(s_{1}, \ldots, s_{k}\right)$ a $\Gamma$-graph manifold, and its metric a $\left(s_{1}, \ldots, s_{k}\right)$-graph metric.

Lemma 4.2. For each $a \in[0,1]^{k}$ the group $\Gamma$ acts freely and isometrically on $M(a)$. For every fixed $q \geq 1$ the assignment $a \in[0,1]^{k} \rightarrow \mu_{q}(M(a))$ is continuous.

Proof. Every element of $\Gamma$ acts on the Cayley graph $G$ by an automorphism which permutes the edges with a given label. For each $a \in[0,1]^{k}$ this automorphism induces an isometry of our manifold $M(a)$ which permutes our copies of $X$ and preserves each of the $k$ collections of hypersurfaces corresponding to one of our generators or its inverse. Since the action of $\Gamma$ on $G$ is free the same is true for the action of $\Gamma$ on $M(a)$. Continuity of the assignment $a \in[0,1]^{k} \rightarrow \mu_{q}(M(a))$ is immediate from Corollary 2.6.

Let again $\Gamma$ be an admissible finite group with an admissible set $\gamma_{1}, \ldots, \gamma_{2 k}$ of generators and corresponding Cayley graph $G$. By definition, this set of generators is minimal with the property that it consists of elements of order at least 3 and contains with each element its inverse. Thus if we fix some $i \leq 2 k$ and if we delete all the edges in $G$ which are either labeled by $\gamma_{i}$ or by $\gamma_{i}^{-1}$ then the resulting graph is disconnected.

Recall that for every complete Riemannian manifold which has eigenvalues below the essential spectrum the multiplicity of the smallest eigenvalue is one. Following the basic idea of $[\mathrm{BC}]$ we use isometric actions of finite groups to construct complete manifolds of bounded curvature with infinitely many eigenvalues below the essential spectrum and such that the multiplicity of the second eigenvalue is bigger than 1 .

Lemma 4.3. Let $\Gamma$ be an admissible group with an admissible set of generators $\gamma_{1}, \ldots, \gamma_{2 k}$. Let $m \geq 2$ be the minimal dimension of a nontrivial irreducible orthogonal representation of $\Gamma$. Then for every $a \in(0,1]^{k}$ which is sufficiently close to 0 , the multiplicity of the second eigenvalue of the $\Gamma$-graph manifold $M(a)$ is at least $m$.

Proof. By our assumption, for each $a \in[0,1]^{k}$ the quotient $M(a) / \Gamma$ is a complete manifold, and the projection $M(a) \rightarrow M(a) / \Gamma$ is a smooth $q$-sheeted covering where $q$ is the cardinality of $\Gamma$. 
The pair $\gamma_{2 i-1}, \gamma_{2 i}$ of generators of $\Gamma$ defines a $\Gamma$-orbit of edges in the Cayley graph $G$. If $s_{i}>0$ then this orbit of edges corresponds to a $\Gamma$-orbit of totally geodesic embedded closed hypersurfaces in $M(a)$ which projects to a closed totally geodesic embedded non-separating hypersurface in $M(a) / \Gamma$. As $s_{i} \searrow 0$ this hypersurface in $M(a) / \Gamma$ degenerates to a pair of cusps.

For each $a \in(0,1]^{k}$ the manifold $M(a)$ is connected. The bottom of the spectrum of $M(a)$ is not contained in the essential spectrum and therefore it is an eigenvalue of multiplicity 1 . The isometric action of $\Gamma$ on $M(a)$ induces an orthogonal representation of $\Gamma$ on the corresponding eigenspace. Since the dimension of this eigenspace is 1 , this representation is trivial and every eigenfunction with respect to this eigenvalue is $\Gamma$-invariant and projects to an eigenfunction on $M(a) / \Gamma$. In particular, the smallest eigenvalue of $M(a)$ coincides with the smallest eigenvalue of $M(a) / \Gamma$.

Now let $a=\left(0, s_{2}, \ldots, s_{k}\right) \in[0,1]^{k}$ where $s_{i}>0$ for $i \geq 2$. By minimality of our set of generators for $\Gamma, M(a)$ consists of at least two isometric components which are permuted by the action of the group $\Gamma$. Thus the multiplicity of the smallest eigenvalue of $M(a)$ (which equals the number of connected components of $M(a)$ ) is at least 2 .

By Theorem A from the introduction, as $s \searrow 0$ the small eigenvalues of $Q(s)=$ $M\left(s, s_{2}, \ldots, s_{k}\right)$ converge to the small eigenvalues of $M(a)$. The multiplicity of the first eigenvalue of $Q(s)$ is 1 and hence for sufficiently small $s$ the second eigenvalue of $Q(s)$ is strictly smaller than the second eigenvalue of $M(a) / \Gamma$. Then an eigenfunction for this eigenvalue can not be $\Gamma$-invariant. This means that the natural orthogonal representation of $\Gamma$ on the eigenspace of $Q(s)$ with respect to the second eigenvalue does not contain a trivial component and the dimension of this eigenspace equals at least the minimal dimension of a nontrivial irreducible orthogonal representation of $\Gamma$. This finishes the proof of our lemma.

We can now iterate this construction as follows. Assume that $\Gamma$ is a finite group which contains a nested sequence $\Gamma>H_{1}>\cdots>H_{m}$ of admissible subgroups $H_{i}$.

Define a set of generators $\gamma_{1}, \ldots, \gamma_{2 k}$ of $\Gamma$ to be $\left(\Gamma, H_{1}, \ldots, H_{m}\right)$-admissible if the following is satisfied.

(1) For every $i \leq m$ there is some $j(i)<k$ such that $\gamma_{1}, \ldots, \gamma_{2 j(i)}$ is an admissible set of generators for $H_{i}$.

(2) For each $i$ the subgroup of $\Gamma$ which is generated by those of our generators which are not contained in $H_{i}$ intersects $H_{i}$ only in the unit element.

We call $\left(\Gamma, H_{1}, \ldots, H_{m}\right)$ an admissible sequence of groups if it admits a $\left(\Gamma, H_{1}, \ldots, H_{m}\right)$-admissible set of generators and if moreover for every $i \geq 1$ the group $H_{i+1}$ is a proper normal subgroup of $H_{i}$. We do not require that $H_{1}$ is a normal subgroup of $\Gamma$.

Now let $\left(\Gamma, H_{1}, \ldots, H_{m}\right)$ be an admissible sequence of groups. For a given choice of a basic manifold $X$ with $2 k$ standard cusps as in Lemma 4.1 we constructed 
above from the Cayley graph of an $\left(\Gamma, H_{1}, \ldots, H_{m}\right)$-admissible set $\gamma_{1}, \ldots, \gamma_{2 k}$ of generators a connected smooth manifold $M$ which admits a natural free action of $\Gamma$ by diffeomorphisms and a natural family of $\Gamma$-invariant metrics. We call our manifold $M$ a $\left(\Gamma, H_{1}, \ldots, H_{m}\right)$-graph manifold.

Corollary 4.4. Let $\left(\Gamma, H_{1}, \ldots, H_{m}\right)$ be an admissible sequence of groups. Let $q \geq 2$ be the minimal dimension of an irreducible orthogonal representation of $\Gamma$ whose restriction to $H_{1}$ is non-trivial. Then there is a family of $\left(\Gamma, H_{1}, \ldots, H_{m}\right)$-graph manifolds for which the multiplicity of the $j$-th eigenvalue for $j=2, \ldots, m$ is at least $q$.

Proof. Let $M$ be a $\left(\Gamma, H_{1}, \ldots, H_{m}\right)$-graph manifold. The group $\Gamma$ acts on $M$ freely as a group of diffeomorphisms and $M \rightarrow M / \Gamma=M_{0}$ is a $|\Gamma|$-sheeted covering. Every complete metric on $M_{0}$ lifts to a $\Gamma$-invariant complete metric on $M$.

Let $\gamma_{1}, \ldots, \gamma_{2 k}$ be a $\left(\Gamma, H_{1}, \ldots, H_{m}\right)$-admissible set of generators for $\Gamma$. Let $\ell<k$ be such that the set $\gamma_{1}, \ldots, \gamma_{2 \ell}$ generates $H_{1}$. Denote by $E$ the subgroup of $\Gamma$ generated by $\gamma_{2 \ell+1}, \ldots, \gamma_{2 k}$. Then $E$ is an admissible finite group which intersects $H_{1}$ trivially. The Cayley graph $G^{\prime}$ of $E$ with respect to the generators $\gamma_{2 \ell+1}, \ldots, \gamma_{2 k}$ is a connected subgraph of $G$. If we remove from $G$ all the edges corresponding to the generators $\gamma_{1}, \ldots, \gamma_{2 \ell}$ then the resulting graph consists of $\left|H_{1}\right|$ disjoint copies of $G^{\prime}$.

Fix some $s_{0}>0$ and for $\left(s_{1}, \ldots, s_{m}\right) \in[0,1]^{m}$ let $Q\left(s_{1}, \ldots, s_{m}\right)$ be the $\Gamma$-graph manifold $M(a)$ where $a=\left(a_{1}, \ldots, a_{k}\right)$ is the $k$-tuple defined as follows: For each $j$ let $i \leq j$ be such that the generator $\gamma_{j}$ is contained in the group $H_{i}$ but not in the group $H_{i+1}$ (where we put $H_{0}=\Gamma$ ) and define $a_{j}=s_{i}$. The group $H_{1}$ acts on $Q\left(s_{1}, \ldots, s_{m}\right)$ as a group of isometries. The manifold $Q(0, \ldots, 0) / H_{1}$ is connected.

Now apply the considerations in the proof of Lemma 4.4 to the graph manifolds $Q(s, 0, \ldots, 0)$. Since the subgroup of $\Gamma$ generated by those elements of $\gamma_{1}, \ldots, \gamma_{2 k}$ which are not contained in $\mathrm{H}_{2}$ intersects $\mathrm{H}_{2}$ trivially, the covering $Q(0, \ldots, 0) / \mathrm{H}_{2}$ of $Q(0, \ldots, 0) / H_{1}$ with deck group $H_{1} / H_{2}$ is disconnected and for $s>0$ the covering $Q(s, 0, \ldots, 0) / H_{2}$ of $Q(s, 0, \ldots, 0) / H_{1}$ with deck group $H_{1} / H_{2}$ is connected. By the considerations in the proof of Lemma 4.4, for sufficiently small $s$ the quotient $Q(s, 0, \ldots, 0) / \mathrm{H}_{2}$ is a complete connected manifold with $\mathrm{H}_{1} / \mathrm{H}_{2}$ invariant metric for which the second eigenvalue is strictly smaller than the second eigenvalue of $Q(s, 0, \ldots, 0) / H_{1}$. Proceeding inductively we obtain in $m-1$ steps a $\left(\Gamma, H_{1}, \ldots, H_{m}\right)$-graph manifold of the form $Q\left(s_{1}, \ldots, s_{m}\right)$ such that the $j$-th eigenvalue for $2 \leq j \leq m$ is strictly smaller than the second eigenvalue of the quotient $Q\left(s_{1}, \ldots, s_{m}\right) / H_{1}$. This then implies that the restriction to $H_{1}$ of the representation of $\Gamma$ on each of the corresponding eigenspaces is nontrivial. This shows the corollary. 
It remains to find admissible finite groups $\Gamma$ with arbitrarily long nested sequences of admissible subgroups $H_{i}$ and for which the smallest dimension of an irreducible representation which is nontrivial on $H_{1}$ is arbitrarily large. This is satisfied for the groups which were already considered by Burger and Colbois [BC].

Namely, let $p \geq 3$ be an odd prime and for some $n \geq 1$ let $\mathbb{F}_{q}$ be the field with $q=p^{n}$ elements and multiplicative group $\mathbb{F}_{q}^{*}=\mathbb{F}_{q}-\{0\}$. For a divisor $r$ of $n$ write $m=\left(p^{n}-1\right) /\left(p^{r}-1\right)$ and define

$$
G_{q, m}=\left\{\left(\begin{array}{cc}
\alpha^{m} & \beta \\
0 & 1
\end{array}\right) \mid \alpha \in \mathbb{F}_{q}^{*}, \beta \in \mathbb{F}_{q}\right\}
$$

Then $G_{q, m}$ is the semi-direct product of $\mathbb{F}_{q}$ with the cyclic group $\mathbb{A}_{q, m}=\left\{a^{m} \mid a \in\right.$ $\left.\mathbb{F}_{q}^{*}\right\}$ of order $p^{r}-1$ which acts on $\mathbb{F}_{q}$ by multiplication. Its commutator subgroup $H_{1}$ is the cyclic group

$$
H_{1}=\left\{\left(\begin{array}{cc}
1 & \beta \\
0 & 1
\end{array}\right) \mid \beta \in \mathbb{F}_{q}\right\}
$$

of order $q$ which can naturally be identified with the additive group $\mathbb{F}_{q}$.

Let $\xi$ be a generator of the cyclic group $\mathbb{F}_{q}^{*}$. Then the dimension of $\mathbb{F}_{q}$ as a vector space over the field $\mathbb{F}_{q}\left[\xi^{m}\right]$ equals $n / r$. Choose a basis $g_{1}, \ldots, g_{n / r} \subset \mathbb{F}_{q}$ for this vector space. For each $i$ the element $g_{i} \in \mathbb{F}_{q}$ generates a cyclic subgroup of $\mathbb{F}_{q} \sim H_{1}$ which is invariant under the action of the group $\mathbb{A}_{q, m}$. The flag of $n / r$ linear subspaces of $\mathbb{F}_{q}$ determined by this basis defines a nested sequence $H_{n / r} \triangleleft \cdots \triangleleft H_{1}$ of normal subgroups of $H_{1}$, and $g_{1}, g_{1}^{-1}, \ldots, g_{n / q}, g_{n / q}^{-1}, \xi^{m}, \xi^{-m}$ is a $\left(G_{q, m}, H_{1}, \ldots, H_{m}\right)$ admissible set of generators for $\Gamma$.

Now since $H_{1}$ equals the commutator of $G_{q, m}$, every character of $G_{q, m}$ (i.e. a one-dimensional unitary representation of $G_{q, m}$ ) factors to a character of $G_{q, m} / H_{1}$. On the other hand, it is well known [M] that the dimension of every irreducible representation of $G_{q, m}$ which is not a character is at least $(q-1) / m$. Thus by Corollary 4.5 the group $G_{q, m}$ gives rise to manifolds for which the $j$-th eigenvalue for $2 \leq j \leq n / r$ has multiplicity at least $(q-1) / m$. Since $n / r$ and $(q-1) / m$ can be chosen arbitrarily large our Theorem $\mathrm{C}$ from the introduction follows.

Acknowledgement. We like to thank the anonymous referee for pointing out a mistake in an earlier version of this paper and for informing us about the references [CV1], [CV2]. The first author is also grateful to Dorothee Schueth for helpful discussions. 
Vol. 80 (2005)

\section{References}

[BC] M. Burger, B. Colbois, A propos de la multiplicité de la première valeur propre d'une surface de Riemann. C. R. Acad. Sci. Paris Sér. I Math. 300 (1985), 247-249. Zbl 0574.53029 MR 0785061

[B] P. Buser, Geometry and spectra of compact Riemann surfaces. Progr. Math. 106, Birkhäuser, Boston, MA, 1992. Zbl 0770.53001 MR 1183224

[BCD] P. Buser, B. Colbois, J. Dodziuk, Tubes and eigenvalues for negatively curved manifolds. J. Geom. Anal. 3 (1993), 1-26. Zbl 0766.58054 MR 1197014

[CT] R. Canary, E. Taylor, Hausdorff dimension and limits of Kleinian groups. Geom. Funct. Anal. 9 (1999), 283-297. Zbl 0935.57021 MR 1692482

[CC1] B. Colbois, G. Courtois, Les valeurs propers inférieures à $\frac{1}{4}$ des surfaces de riemann de petit rayon d'injectivité. Comment. Math. Helv. 64 (1989), 349-362. Zbl 0684.53040 MR 0998853

[CC2] B. Colbois, G. Courtois, Convergence de variétés et convergence du spectre du laplacien. Ann. Sci. École Norm. Sup. (4) 4 (1991), 507-518. Zbl 0754.58040 MR 1123559

[CV1] Y. Colin de Verdière, Sur la multiplicité de la première valeur propre non nulle du laplacien. Comment. Math. Helv. 61 (1986), 254-270. Zbl 0607.53028 MR 0856089

[CV2] Y. Colin de Verdière, Construction de Laplaciens dont une partie finie du spectre est donnée. Ann. Sci. École Norm. Sup. (4) 20 (1987), 599-615. Zbl 0636.58036 MR 0932800

[CoT] T. Comar, E. Taylor, Geometrically convergent Kleinian groups and the lowest eigenvalue of the Laplacian. Indiana Univ. Math. J. 47 (1998), 601-623. Zbl 0916.30040 MR 1647944

[D] E. B. Davies, Spectral theory and differential operators. Cambridge Stud. Adv. Math. 42, Cambridge University Press, Cambridge 1995. Zbl 0893.47004 MR 1349825

[F] K. Fissmer, Diskretes Spektrum von hyperbolischen Flächen: Beispiele für Konvergenz. Diplomarbeit, Bonn 2000.

[G] M. Gromov, Metric structures for Riemannian and non-Riemannian spaces. Progr. Math. 152, Birkhäuser, Boston, MA, 1999. Zbl 0953.53002 MR 1699320

[H] U. Hamenstädt, Small eigenvalues of geometrically finite manifolds. J. Geom. Anal. 14 (2004), 281-290. Zbl 02147102 MR 2051688

$[\mathrm{dH}]$ P. de la Harpe, Topics in geometric group theory. Chicago Lectures in Mathematics, University of Chicago Press, Chicago, IL, 2000. Zbl 0965.20025 MR 1786869

[J] C. Judge, Tracking eigenvalues to the frontier of moduli space I: Convergence and spectral accumulation. J. Funct. Anal. 184 (2001), 273-290. Zbl 1005.58012 MR 1850999

[M] G. W. Mackey, Induced representations of groups and quantum mechanics. W. A. Benjamin Inc., New York, Amsterdam; Editore Boringhieri, Turin, 1968. Zbl 0174.28101 MR 0507212

[MM] C. McMullen, Hausdorff dimension and conformal dynamics I: Strong convergence of Kleinian groups. J. Differential Geom. 51 (1999), 471-515. Zbl 1023.37028 MR 1726737 
[T] E. Taylor, Geometric finiteness and the convergence of Kleinian groups. Comm. Anal. Geom. 5 (1997), 497-533. Zbl 0896.20033 MR 1487726

Received May 13, 2002; revised November 3, 2003

Karsten Fissmer, Perthesanlage 7a, 53119 Bonn, Germany

Ursula Hamenstädt, Mathematisches Institut der Universität, Beringstraße 1, 53115 Bonn,

Germany

E-mail: ursula@math.uni-bonn.de 